\title{
COMPACTIFICATIONS OF HARMONIC SPACES
}

\author{
C. Constantinescu and A. Cornea
}

Many results of the theory of Riemann surfaces derive only from the properties of the sheaf of harmonic functions on these surfaces. It is therefore natural to try to extend these results to more comprehensive structures defined by means of a sheaf of continuous functions on a topological space which should possess the main properties of the sheaf of harmonic functions on a Riemann surface. The aim of the present paper is to generalise some known results from the theory of Riemann surfaces to spaces endowed with sheaves satisfying Brelot's axioms [2], which we call harmonic spaces. In order to do so we had to introduce and to study the maps, associated in a natural way with this structure, called harmonic maps; they replace the analytic maps between Riemann surfaces. In this general frame we reconstruct the whole theory of Wiener compactification as well as the theory of the behaviour of analytic maps at the Wiener boundary.

The first paragraph contains some simple remarks about the Dirichlet problem which could not be found in the existing literature. In the second paragraph we introduce and study the operator $h$ and the Wiener functions which represent the main tool of the present paper. The harmonic maps are studied in the third paragraph. In the fourth paragraph we treat some problems concerning general compactifications and in the fifth one the particular case of Wiener compactification is considered. This compactification is closely related to Feller's ideal boundary. The last paragraph is devoted to the problem of the behaviour of the harmonic maps at the Wiener boundary.

Without mentioning the source we have borrowed intensively ideas, methods and usual tricks from various papers on the theory of Riemann surfaces. For many of them we are indebted to K. Hayashi, M. Heins, Y. Kusunoki, S. Mori, M. Nakai. A detailed bibliography in this direction may be found in our book “Ideale Ränder Riemannscher Flächen”, Springer Verlag, 1963.

Received December 3, 1963. 


\section{$\S$ 1. Preliminaries}

1.1. We call a harmonic space a locally compact space ${ }^{*)}$ on which it is given a sheaf of continuous functions, called harmonic functions, satisfying the axioms 1, 2, 3 from [2]. We denote by $\mathfrak{P}$ (resp. $\mathfrak{S}_{\text {) }}$ the class of harmonic spaces on which there exists a positive potential (resp. a positive harmonic function).

We shall denote in this paragraph by $X$ a harmonic space on which there exists a positive superharmonic function, i.e. $X \in \mathfrak{B} \cup \mathfrak{S}$. For any open subset $U$ of $X$ we denote by $\partial U$ the boundary of $U$. Let $U$ be an open subset of $X$, $U \in \mathfrak{P}$. For any real function $f^{* *)}$ defined on a set containing $\partial U$ we denote by $\overline{\mathscr{S}}_{f}^{U, x}=\overline{\mathscr{S}}_{f}^{I}=\overline{\mathscr{Y}}_{f}$ (resp. $\mathscr{\mathscr { L }}_{f}^{T, x}=\mathscr{\mathscr { L }}_{f}^{U}=\mathscr{\mathscr { S }}_{f}$ ) the set of lower bounded hyper: harmonic (resp. upper bounded hypoharmonic) functions $s$ on $U$ such that non-negative (resp. non-positive) outside a compact set $K_{s}$ of $X$ and for any point $y \in \partial U$

$$
\left.\liminf _{x \rightarrow y} s(x) \geq f(y) \quad \text { (resp. } \limsup _{x \rightarrow y} s(x) \leq f(y)\right) .
$$

Any function of $\overline{\mathscr{S}}_{f}^{U, x}$ (resp. $\mathscr{S}_{f}^{U, x}$ ) possesses a non-positive subharmonic minorant (resp. non-negative superharmonic majorant). If $\bar{s} \in \overline{\mathscr{S}}_{f}^{v}, x, \underline{s} \in \underline{\mathscr{S}}_{f}^{u}, x$ then $\underline{s} \leq \bar{s}$. This follows for the non-compact components of $U$ from Corollary 1 in [3] and for a compact component of $U$ (Theorem 6 of [3]). We denote by $\bar{H}_{f}^{r}, x=\bar{H}_{f}^{U}=\bar{H}_{f}$ (resp. $\underline{H}_{f}^{\tau,}, x=\underline{H}_{f}^{U}=\underline{H}_{f}$ ) the greatest lower bound of $\overline{\mathscr{S}}_{f}^{U, x}$ (resp. the least upper bound of $\mathscr{S}_{f}^{U, x}$ ). The restrictions of $\bar{H}_{f}^{U, x}, \underline{H}_{f}^{v}, x$ to any component of $U$ are either differences of non-negative harmonic functions or identically $\pm \infty$ and we have $\underline{H}_{f}^{u, x} \leq \bar{H}_{f}^{u, x}$. The function $f$ is called resolutive (with respect to $(U, X))$ if $\bar{H}_{f}^{v, x}$ and $\underline{H}_{f}^{i, x}$ are finite and equal; in that case we denote by $H_{f}^{U, x}=H_{f}^{l ;}=H_{f}$ their common value.

Lemma 1.1. Let $U, U^{\prime}$ be open subsets of $X, U \subset U^{\prime}, U \in \mathfrak{P}$, and let $f$ be $a$ real function on $U^{\prime} \cap \partial U$. If we denote by $f_{0}$ the function on $\partial U$ equal to $f$ on $U^{\prime} \cap \partial U$ and equal to 0 on $\partial U-U^{\prime}$ we have

$$
\bar{H}_{f}^{U,,^{\prime \prime}}=\bar{H}_{f_{0}}^{U, x}, \quad \underline{H}_{f}^{U, U^{\prime}}=\underline{H}_{f_{0}}^{l, x} .
$$

Obviously $\overline{\mathscr{S}}_{f}^{U, U^{\prime}} \subset_{c} \overline{\mathscr{S}}_{f_{0}^{\prime}}^{v^{\prime} x}$ and therefore $\bar{H}_{f_{0}}^{U, x} \leq \bar{H}_{f}^{U, U^{\prime}}$. Let $s_{0}$ be a positive superharmonic function on $X$. For any $\bar{s} \in \overline{\mathscr{S}}_{f_{0}}^{U, X}$ and $\varepsilon>0$ we have $\bar{s}+\varepsilon s_{0} \in \overline{\mathscr{S}}_{f}^{\pi,}, v^{\prime}$.

*) We don't require that the space is non-compact and connected.

**) $f$ may take the values $\pm \infty$. 
Hence

$$
\bar{H}_{f}^{U, U^{\prime}} \leq \bar{s}+\varepsilon S_{0} .
$$

$\varepsilon$ and $\bar{s}$ being arbitrary we get $\bar{H}_{f}^{U, U^{\prime}} \leq \bar{H}_{f_{0}}^{i, x}$.

Lemma 1.2. Let $U, V$ be open subsets of $X, V \subset U \in \mathfrak{B}$, and let $f$ be a real function on $\partial U$. If we denote by $f^{\prime}$ the function on $\bar{U}$ equal to $f$ on $\partial U$ and equal to $\bar{H}_{f}^{u, x}$ on $U$ then

$$
\bar{H}_{f}^{U, x}=\bar{H}_{f}^{v, x}
$$

on $V$.

We may suppose $U$ is connected. Let $s \in \overline{\mathscr{S}}_{f}^{U, x}$. Then $s=\overline{\mathscr{S}}_{f}^{r}, x$ and therefore $\bar{H}_{f}^{V}{ }^{\prime} x \leq \bar{H}_{f}^{u, x}$. If $\bar{H}_{f} \cdot x=-\infty$ the required equality is obvious.

Suppose now $\bar{H}_{f}^{r, x}$ finite. Let $s \in \overline{\mathscr{S}}_{f}^{r, x}, s^{\prime} \in \overline{\mathscr{S}}_{f}^{r}{ }^{r}$. The function on $U$ equal to $s$ on $U-V$ and equal to $s-\bar{H}_{f}^{U, x}+\min \left(\bar{H}_{f}^{U, x}, s^{\prime}\right)$ on $V$ is hyperharmonic and belongs to $\overline{\mathscr{S}}_{f}^{U, x}$. Hence

$$
\bar{H}_{f}^{u, x} \leq s-\bar{H}_{f}^{U, x}+s^{\prime} .
$$

$s$ and $s^{\prime}$ being arbitrary we get $\bar{H}_{f}^{r, x} \leq \bar{H}_{f^{\prime}}^{v, x}$.

If $\bar{H}_{f}^{r, x}=+\infty$ then for any $s^{\prime} \in \overline{\mathscr{S}}_{f^{\prime}}^{r, x}$ the function equal to $+\infty$ on $U-V$ and equal to $s^{\prime}$ on $V$ belongs to $\overline{\mathscr{S}}_{f^{\prime}}^{U}{ }^{x}$. Hence $s^{\prime}=+\infty$ and $\bar{H}_{f^{\prime}}^{v}{ }^{x}=+\infty$.

Theorem 1.1. Let $U$ be an open subset of $X, U \in \mathfrak{P}$. For any non-negative Borel-function $f$ on $\partial U$ we have

$$
\bar{H}_{f}^{U, x}=\underline{H}_{f}^{U, x} .
$$

Let $s$ be a finite positive continuous superharmonic function on $X$. From Corollary 3 in [1] it follows that for any natural number $\boldsymbol{n}$ the function $\min (f, n s)$ is resolutive. Hence

$$
\bar{H}_{f}=\lim _{n \rightarrow \infty} H_{\min (f, n s)} \leq \underline{H}_{f} \leq \bar{H}_{f} .
$$

CoRollary 1.1. If $s$ is a non-negative superharmonic function on $X$ and $U$ an open subset of $X, U \in \mathfrak{P}$, then

$$
H_{s}^{(j, x}=R_{s}^{X-U *)}
$$

on $U$.

*) See [2], page 80 , definition 9 . 
Obviously $H_{s}^{U, x} \leq R_{s}^{X-U}$. Let $\bar{s} \in \overline{\mathscr{S}}_{s}^{U, x}$. The function on $X$ equal to $s$ on $X-U$ and equal to $\min (s, \bar{s})$ on $U$ is a non-negative superharmonic function. It follows $R_{s}^{X-U} \leq \bar{s}, R_{s}^{X-U} \leq H_{s}^{U, X}$ on $U$.

Lemma 1.3. Let $U$ be an open subset of $X, U \in \mathfrak{P}$, and let $f$ be a nonnegative real function on $\partial U$ such that $\bar{H}_{f}^{U, x}$ is finite. If $u$ is a continuous function on $\bar{U}$, harmonic on $U,|u| \leq \bar{H}_{f}^{U, x}$ on $U$, then $u$ is resolutive and

$$
\boldsymbol{u}=H_{u}^{U, x} .
$$

Since

$$
\underline{H}_{-f} \leq \underline{H}_{u} \leq \bar{H}_{u} \leq \bar{H}_{f},
$$

$u$ is resolutive by Theorem 1.1 .

Let $s \in \overline{\mathscr{S}}_{f}$. Then $s-u \in \overline{\mathscr{S}}_{f-u}$ and therefore

$$
\bar{H}_{f}=\bar{H}_{(f-u)+u} \leq \bar{H}_{f-u}+H_{u} \leq s-u+H_{u} .
$$

$s$ being arbitrary we get

$$
\bar{H}_{f} \leq \bar{H}_{f}-u+H_{u}, \quad u \leq H_{u} .
$$

Similarly we deduce $u \geq H_{u}$.

1.2. A potential $p$ on a non-compact connected space $X$ is called an Evans potential if there exists a positive potential $p^{\prime}$ on $X$ such that $\frac{p}{p^{\prime}}$ converges to infinite at the Alexandroff point of $X$ (here $\frac{\infty}{\infty}=\infty$.) $p$ is called also an Evans potential associated with $p^{\prime}$.

Lемма 1.4. If $X$ is connected and non-compact, then for any positive potential $p^{\prime}$ there exists an Evans potential associated with $p^{\prime}$, continuous if $p^{\prime}$ is continuous and finite.

The existence of an Evans potential $p$ associated with $p^{\prime}$ was proved in Lemma 1 in [1]. Suppose now $p^{\prime}$ continuous. Let $X^{*}$ be the Alexandroff compactification of $X$. The function equal to $\frac{p}{p^{\prime}}$ on $X$ and equal to $\infty$ at the Alexandroff point of $X$ is lower semi-continuous. There exists therefore a continuous non-negative function $f$ on $X^{*}$ equal to $\infty$ at the Alexandroff point of $X$ and smaller than $\frac{p}{p^{\prime}}$ on $X . \hat{R}_{f p^{\prime}}^{X}$ is a continuous superharmonic function Theorem 3 in [3]. Since it is dominated by $p$ it is a potential and since it domi- 
nates $f p^{\prime}$ it is an Evans potential associated with $p^{\prime}$.

1.3. Let $U$ be an open subset of $X, f$ be a real function on $U$ and $x \in \partial U$. We say that $f$ is associated with zero at $x$ if there exists a regular neighbourhood $V$ of $x$ and a positive superharmonic function s defined on a neighbourhood of $\bar{V}$ such that $|f| \leq H_{s}^{r i n} r^{r}$, . If $f$ is harmonic on the trace on $U$ of a neighbourhood of $x$ then $f$ is associated with zero at $x$ if and only if there exists a regular neighbourhood $W$ of $x$ such that $f$ is bounded on $U \cap \bar{W}$ and $f=H_{f}^{U \cap W, v}$. Indeed if $f$ is associated with zero at $x$, then $f$ is bounded on a neighbourhood of $x$ since $H_{s}^{U \cap r^{r}, U} \leq H_{s}^{r, x}$ and by Lemma 1.3 and Lemma 1.2, $f=H_{f}^{U \cap W, U^{r}}$ for any $\bar{W} \subset V$. The converse is trivial.

Lemma 1.5. Let $U$ be a domain on $X, U \in \mathfrak{P}$, and $u$ a harmonic function on $U$ associated with zero at a boundary point $y$ of $U$. For any Evans potential $p$ on $U$ we have

$$
\lim _{x \rightarrow y} \inf (u(x)+p(x)) \geq 0 .
$$

Let $V$ be a regular neighbourhood of $y$ such that $u=H_{u}^{U n v, v}$ and $u$ is bounded on $U \cap V$, and let $f$ be the function on $\partial(U \cap V)$ equal to $|u|$ on $U \cap \partial V$ and equal to 0 on $\bar{V} \cap \partial U$. Then $|u| \leq H_{f}^{U \cap} \cap r^{r}, x$.

Let $p^{\prime}$ be a positive potential on $U$ such that $p$ is an Evans potential associated with $p^{\prime}$ and let $\mathfrak{u}$ be an ultra-filter on $U$ converging to $y$. If $p^{\prime}$ converges to zero along $\mathfrak{u}$ then by Lemma 2 in [1]

$$
0 \leq \lim _{\mathfrak{u}}|u| \leq \lim _{\mathfrak{u}} H_{f}^{U \cap r^{r}, x}=0, \quad \lim _{\mathfrak{u}}(u+p) \geq 0 .
$$

If $p^{\prime}$ doesn't converge to zero along $\mathfrak{U}$ then $p$ converges to infinite along $\mathfrak{l}$ and

$$
\lim _{\mathfrak{u}}(u+p) \geq 0 \text {. }
$$

THEOREM 1.2. Let $U \in \mathfrak{P}$ be an open set and $u$ be a non-negative harmonic function on $U$ associated with zero at any boundary point of $U$. The function $s$ on $X$ equal to $u$ on $U$ and equal to 0 on $X-U$ is nearly subharmonic and the least upper bound of the family of its continous subharmonic minorants.

Let $V$ be a regular domain, $U_{c}$ be a component of $U$ and $p_{c}$ be an Evans potential on $U_{l}$. The function $H_{s}^{V, x}+\varepsilon p_{\iota}-u$ is superharmonic on $V \cap U_{c}$ for $\varepsilon>0$ and for any boundary point $y$

$$
\liminf _{x \rightarrow y}\left(H_{s}^{r, x}(x)+\varepsilon p_{\iota}(x)-u(x)\right) \geq 0
$$


This is obvious for $y \in U_{\imath} \cap \partial V$ and for $y \in \bar{V} \cap \partial U_{\iota}$ it follows from the preceding lemma $\varepsilon p_{c}$ being an Evans potential. Hence $H_{s}^{r, x}+\varepsilon p_{\iota}-u$ is non-negative. $\varepsilon$ being arbitrary we get

$$
u \leq H_{s}^{r, x}
$$

on $V \cap U_{\iota}$. $U_{\iota}$ being arbitrary and $s$ equal to 0 on $X-U$ it follows

$$
s \leq H_{s}^{v, x}
$$

on $V$.

For any $\varepsilon>0$ and any finite system $\left\{U_{\iota_{1}}, \ldots, U_{\iota_{n}}\right\}$ of components of $U$ we denote by $s_{\varepsilon \ell_{1} \ldots \iota_{n}}$ the function on $X$ equal to 0 on $X-\bigcap_{j=1}^{n} U_{\ell_{j}}$ and equal to $\max \left(u-\varepsilon p_{\ell j}, 0\right)$ on $U_{\imath j}$, where $p_{\iota_{j}}$ is a continuous Evans potential on $U$. It is easy to see that the functions $s_{\varepsilon \ell_{1} \ldots \iota_{n}}$ are subharmonic and continuous and $s$ is their least upper bound.

\section{§. Harmonizable functions}

2.1. In this paragraph $X$ denotes a harmonic space on which there exists a positive superharmonic function, i.e. $X \in \mathfrak{P} \cup \mathfrak{S}$.

Let $U$ be an open subset of $X, U \in \mathfrak{P}$, and $f$ be a real function defined on a set containing $U-K$, where $K$ is a compact subset of $X$. We denote by $\overline{\mathscr{W}}_{f}^{U}, x=\overline{\mathscr{W}}_{f}^{U}=\overline{\mathscr{W}}_{f}\left(\right.$ resp. $\left.\mathscr{\mathscr { P }}_{f}^{U, x}=\mathscr{\mathscr { W }}_{f}^{U}=\mathscr{W}_{f}\right)$ the set of hyperharmonic (resp. hypoharmonic) functions $s$ on $U$ such that: a) $s$ possesses a non-positive subharmonic minorant (resp. a non-negative superharmonic majorant); b) $s$ dominates (resp. is dominated by) $f$ outside a compact subset $K_{s}$ of $X$; c) for any $y \in \partial U$

$$
\liminf _{x \rightarrow y} s(x) \geq 0 \quad \text { (resp. } \limsup _{x \rightarrow y} s(x) \leq 0 \text { ). }
$$

It follows from the definition that $\overline{\mathscr{W}}_{f}^{I r}, x$, $\underline{\mathscr{P}}_{f}^{T, x}$ depend only on the values taken by $f$ in a neighbourhood of the Alexandroff point of $X$. If $f$ and $f^{\prime}$ coincide outside a compact subset of $X$ we have $\overline{\mathscr{W}}_{f}^{\pi \cdot x}=\overline{\mathscr{W}}_{f}^{\tau}, x, \underline{\mathscr{W}}_{f}^{U, x}=\mathscr{\mathscr { P }}_{f}^{U, x}$. If $\bar{s} \in \overline{\mathscr{W}}_{f}^{U, x}$, $\underline{s} \in \mathscr{L}_{f}^{U}, x$ then $\underline{s} \leq \bar{s}$. This follows for a non-compact component of $U$ from Corollary 1 in [3] and for a compact component of $U$ from Theorem 6 in [3]. We denote by $\bar{h}_{f}^{v, x}=\bar{h}_{f}^{t^{r}}=\bar{h}_{f}$ (resp. $\underline{h}_{f}^{u, x}=\underline{h}_{f}^{u}=\underline{h}_{f}$ ) the greatest lower bound of $\bar{y}_{f}^{r}, x$ (resp. the least upper bound of $\underline{M}_{f}^{U}, x$ ). Obviously $\underline{h}_{f}^{U, x} \leq \bar{h}_{f}^{T, x}$ and their restrictions to any component of $U$ are either differences of non-negative harmonic functions or identically $\pm \infty$. If $U^{\prime}$ is a component of $U$ and $X^{\prime}$ the 
component of $X$ containing $U^{\prime}$ then $\bar{h}_{f}^{U^{\prime}, x^{\prime}}=\bar{h}_{f}^{U, x}, \underline{h}_{f}^{U^{\prime}, x^{\prime}}=\underline{h}_{f}^{U, x}$.

If $s$ is a non-negative superharmonic function on $X, \bar{h}_{s}^{G, X}$ is associated with zero at any boundary point of $U$ and for $U=X$ it is the greatest harmonic minorant of $s$. For the first assertion it is sufficient to see that for any rela-

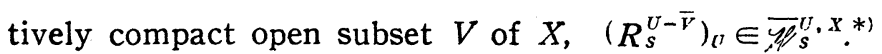

Lemma 2.1. Let $U$ be an open subset of $X, U \in \mathfrak{P}$, and let $f, f^{\prime}$ be real functions defined on $U$ outside a compact subset of $X$ and $\alpha$ be a positive number. We have:

a) $\bar{h}_{-f}=-\underline{h}_{f}$;

b) $\bar{h}_{\alpha f}=\alpha \bar{h}_{f}, \underline{h}_{\alpha f}=\alpha \underline{h}_{f}$;

c) $\bar{h}_{f+f^{\prime}} \leq \bar{h}_{f}+\bar{h}_{f^{\prime}}, \underline{h}_{f+f^{\prime}} \geq \underline{h}_{f}+\underline{h}_{f^{\prime}}{ }^{* *)}$, whenever the second part of the inequalities has a sense;

d) if $f \leq f^{\prime}$ outside a compact subset of $X$ then $\bar{h}_{f} \leq \bar{h}_{f^{\prime}}, \underline{h}_{f} \leq \underline{h}_{f^{\prime}}$;

e) if $\ddot{h}_{f}, \bar{h}_{f^{\prime}}\left(\right.$ resp. $\left.\underline{h}_{f}, \underline{h}_{f^{\prime}}\right)$ are finite then

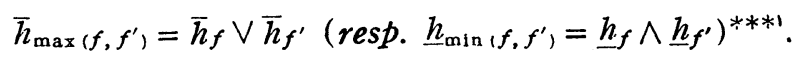

The only non-trivial relation is

$$
\bar{h}_{\max \left(f, f^{\prime}\right)} \leq \bar{h}_{f} \vee \bar{h}_{f^{\prime}} .
$$

Let $s \in \overline{\mathscr{W}}_{f}, s^{\prime} \in \overline{\mathscr{W}}_{f^{\prime}}$. Then $\bar{h}_{f} \vee \bar{h}_{f^{\prime}}+s-\bar{h}_{f}+s^{\prime}-\bar{h}_{f^{\prime}} \in \overline{\mathscr{Y}}_{\max }\left(f . f^{\prime}\right)$ and the inequality is proved $s$ and $s^{\prime}$ being arbitrary.

Lemma 2.2. Let $U$ be a domain on $X, U \in \Re$, and $f$ be a real function defined on $U$ outside a compact subset of $X$. If $\bar{h}_{f}^{r}, x$ is finite then there exists a non-negative superharmonic function $s$ on $U$ such that for any $\varepsilon>0 \bar{h}_{f}^{r, x}+\varepsilon s$ $\in \overline{\mathscr{V}}_{f}^{U, X}$. If $U=X$ then there exists a potential $s$ with the same property.

Let $\left\{s_{n}\right\}$ be a sequence of $\overline{\mathscr{P}}_{f}$ such that

$$
s=\sum_{n=1}^{\infty}\left(s_{n}-\bar{h}_{f}\right)
$$

is convergent at a point. This function fulfils the conditions of the lemma.

Suppose now $U=X$. Let $K_{n}$ be a compact set such that $s_{n} \geq f$ on $X-K_{n}$.

*) See [2], page 82 , definition 10 .

**) Here $f+f^{\prime}$ is defined arbitrarily if the operation has no sense.

$* * *$ ) If $u, v$ are differences of non-negative harmonic functions $u \vee v$ (resp. $u \wedge v$ ) denotes the least harmonic majorant (resp. the greatest harmonic minorant) of $u$ and $v$. 
Let $\left\{X_{n}\right\}$ be a pseudo-exhaustion of $X$ (See Theorem 8 in [3]) such that $X_{n} \supset K_{j}$ for $j \leq 2 n . \quad p_{n}=R_{s_{n}-h_{f}}^{X_{n}}$ is a potential (Proposition 10 in [2]). It follows that

$$
\sum_{n=1}^{\infty} p_{n}
$$

is also a potential (Lemma 3, [3]). There exists a potential $p_{0}$ infinite on $X-\bigcup_{n=1}^{\infty} X_{n}$ by Theorem 9 in [3]. We denote

$$
p=p_{0}+\sum_{n=1}^{\infty} p_{n}
$$

$p$ is a potential and we have for $\varepsilon>0, m \geq \frac{1}{\varepsilon}, j \geq 1$ and $x \in X_{m+2}-X_{m+j}$

$$
\bar{h}_{f}(x)+\varepsilon p(x) \geq \bar{h}_{f}(x)+\frac{1}{m} \sum_{n=m+2 j}^{2 m+2 j} p_{n}(x)=\frac{1}{m} \sum_{n=m+2 j}^{2 m+2 j} s_{n}(x) \geq f(x) .
$$

It follows immediately

$$
\bar{h}_{f}+\varepsilon p \geq f
$$

on $X-X_{m+1}$.

We denote by $(B$ the set of open relatively compact sets of $X$. For any $U \in \mathbb{B}$ we denote

$$
\mathbb{B}_{U}=\left\{U^{\prime} \in \mathbb{B} \mid U^{\prime} \supset U\right\} .
$$

$\left\{\mathbb{S}_{U}\right\}_{U \in \mathbb{S}}$ is a basis of a filter on $\mathbb{B}$. We denote this filter by $\mathbb{B}^{*}$.

LEMMA 2.3. Let $U$ be an open subset of $X, U \in \mathfrak{B}$, and $f$ be a real function on $X$. Then

$$
\underline{h}_{f}^{U} \cdot x \leq \lim _{V, \mathbb{S}^{*}} \underline{H}_{f}^{U \cap r, v} \leq \underset{V, \mathbb{S}^{*}}{\lim \sup _{f}} \bar{H}_{f}^{U \cap v, v} \leq \bar{h}_{f}^{U, x} .
$$

Let $s \in \overline{\mathscr{W}}_{f}^{U, x}$. There exists $V \in \mathbb{S}$ such that $s \geq f$ on $U-V$. For any $V^{\prime} \in \mathbb{S}_{V} s \in \overline{\mathscr{S}}_{f_{0}}^{U \cap \nu^{\prime}, x}$, where $f_{0}$ is the function on $X$ equal to $f$ on $U$ and. equal to 0 on $X-U$. Hence by Lemma 1.1

$$
\bar{H}_{f}^{U \cap V^{r}, U}=\bar{H}_{f_{0}}^{U \cap r^{*}, X} \leq s, \quad \underset{V, \mathbb{S}^{*}}{\lim \sup _{f}} \bar{H}_{f}^{U \cap r^{r}, U} \leq s, \quad \lim _{V, \mathbb{S}^{*}} \sup _{f} \bar{H}_{f}^{U \cap V, U} \leq \bar{h}_{f} .
$$

Lemma 2.4. Let $U$ be an open subset of $X, U \in \mathfrak{P}$, and $f$ be a non-negative function on $\partial U$. If $u=\bar{H}_{f}^{U, x}$ is finite then $\bar{h}_{u}^{U, x}=0$.

We may suppose $U$ connected and $f$ lower semi-continuous. For any $\underline{s} \in \underline{\mathscr{S}}_{f}^{c}, x, u-\underline{s} \in \overline{\mathscr{W}}^{c} u$. Hence 


$$
\bar{h}_{u}^{U, x} \leq u-\underline{s} .
$$

$\underline{s}$ being arbitrary, $\bar{h}_{u}^{U, x}$ vanishes by Theorem 1.1 .

Lemma 2.5. Let $U, U^{\prime}$ be open subsets of $X, U \subset U^{\prime} \in \mathfrak{P}$, and $f$ be a real function defined on $U^{\prime}$ outside a compact subset of $X$. If $f^{\prime}=\bar{h}_{f}^{U^{\prime}, X}$ is finite then $\bar{h}_{f}^{U} \cdot x=\bar{h}_{f}^{U}, x$.

If $s \in \overline{\mathscr{P}}_{f}^{U, x}$ and $s^{\prime} \in \overline{\mathscr{P}}_{f}^{U^{\prime}}, x$ then $s+s^{\prime}-f^{\prime} \in \overline{\mathscr{P}}_{f}^{U, x}$. Hence

$$
\bar{h}_{f}^{U, x} \leq s+s^{\prime}-f^{\prime}, \quad \bar{h}_{f}^{U, x} \leq \bar{h}_{f}^{U, x} .
$$

In order to prove the converse inequality we suppose $U^{\prime}$ connected. Let $s^{\prime} \in \overline{\mathscr{W}}_{f}^{U^{\prime}, x}, s^{\prime}$ superharmonic, $s \in \overline{\mathscr{W}}_{f}^{U, x}, \bar{s} \in \overline{\mathscr{S}}_{\max \left(s^{\prime}, 0\right)}^{U, U^{\prime}}$. The function on $U^{\prime}$ equal to $s^{\prime}$ on $U^{\prime}-U$ and equal to $\min \left(s^{\prime}, s+\bar{s}\right)$ on $U$ is superharmonic and belongs to $\overline{\mathscr{Y}}_{f}^{U^{\prime}}, x$. Hence $f^{\prime} \leq s+\bar{s}$ on $U$. Since $\bar{s}$ is arbitrary we get $f^{\prime} \leq s+\bar{H}_{\max \left(s^{\prime}, 0\right)}^{U, U^{\prime}}$. By definition there exists a non-positive subharmonic minorant $\underline{s}$ of $s^{\prime}$. Since $\boldsymbol{s}^{\prime}-\underline{s}$ is a superharmonic function belonging to $\overline{\mathscr{S}}_{\max \left(S^{\prime}, 0\right),}^{U, U^{\prime}} \bar{H}_{\max \left(s^{\prime}, 0\right)}^{U, U^{\prime}}$ is finite. By Lemma 2.4 and Lemma 2.2 there exists a non-negative superharmonic function $s_{0}$ on $U$ such that for any $\varepsilon>0 \varepsilon s_{0} \geq \bar{H}_{\max \left(s^{\prime}, 0\right)}^{\left(;, U^{\prime}\right.}$ on $U$ outside a compact set of $U^{\prime}$. Then $s+\varepsilon s_{0} \in \overline{\mathscr{W}}_{f^{\prime}}^{U, x}$ and we have, $\varepsilon$ and $s$ being arbitrary, $\bar{h}_{f^{\prime}}^{U}, x \in \bar{h}_{f}^{f}, x$.

Lemma 2.6. Let $X \in \mathfrak{P}, A \subset X$ and $f$ be a real function defined on $X$ outside a compact subset of $X$, such that $\underline{h}_{f}^{X}$ is finite and non-negative and $f$ is non-positive quasi everywhere ${ }^{* 1}$ on $A$ outside a compact subset of $X$. If we denote $u=\underline{h}_{f}^{X}$ then $\hat{R}_{u}^{A * *)}$ is a potential.

Let $p$ be a potential such that $u-p \in \mathscr{W}_{f}$ (Lemma 2.2). There exists a compact set $K$ such that $u-p \leq f$ on $X-K$ and $f \leq 0$ quasi-everywhere on $A-K$. It follows

$$
\hat{R}_{u}^{A} \leq \hat{R}_{u}^{K}+p .
$$

Lemma 2.7. Let $U$ be an open set, $U \in \mathfrak{P}$, and $f$ be a continuous finite function on $\bar{U}$. If $\bar{h}_{f}^{U, U}$ or $\underline{h}_{f}^{U}, U$ or both functions $\bar{h}_{f}^{U, x}, \underline{h}_{f}^{U, x}$ are finite, $f$ is resolutive with respect to $(U, X)$. If $f$ is resolutive with respect to $(U, X)$ then

$$
\bar{h}_{f}^{v, v}=\bar{h}_{f}^{U, x}+H_{f}^{U, x}, \quad \underline{h}_{f}^{U, v}=\underline{h}_{f}^{U, x}+H_{f}^{U, x} .
$$

Suppose $\bar{h}_{f}^{G, U}$ does not take the value $+\infty$. Let $s \in \overline{\mathscr{W}}_{f}^{U, t^{r}}$ and $\underline{s}$ be a non.

*) See [2], page 124, definition 21.

**) See [2], page 80, definition 9. 
positive subharmonic minorant of $s$. Then $s-\underline{s} \in \overline{\mathscr{V}}_{f}^{W}, x$ and $\bar{h}_{f}^{W, x}$ does not take the value $+\infty$. Similarly if $\underline{h}_{f}^{U}$, does not take the value $-\infty$ then $\underline{h}_{f}^{U}$, $x$ does not take the value $-\infty$.

Suppose now $\bar{h}_{f}^{U, x}$ does not take the value $+\infty$. Let $s \in \overline{\mathscr{W}}_{f}^{U}, x, \underline{s}$ be a nonpositive subharmonic minorant of $s$ and $s_{0}$ be a positive superharmonic function on $X$. Since there exists a compact set $K$ on $X$ such that $s \geq f$ on $U-K$ there exists a positive number $\alpha$ such that $s-\underline{s}+\alpha s_{0} \in_{c} \overline{\mathscr{S}}_{f}^{U}, x$. It follows that $\bar{H}_{f}^{U, x}$ does not take the value $+\infty$. Let $s_{1} \in \overline{\mathscr{V}}_{f}^{U, x}, s_{2} \in \overline{\mathscr{S}}_{f}^{v, x}$. For any $\varepsilon>0, s_{1}+s_{2}+\varepsilon s_{0} \in \overline{\mathscr{W}}_{f}^{U} \cdot U$ and therefore

$$
\bar{h}_{f}^{-U, U} \leq s_{1}+s_{2}+\varepsilon s_{0} .
$$

$s_{1}, s_{2}$ and $\varepsilon$ being arbitrary we get

$$
\bar{h}_{f}^{U, U} \leq \bar{h}_{f}^{U, x}+\bar{H}_{f}^{U, x} .
$$

Similarly if $\underline{h}_{f}^{r}, x$ does not take the value $-\infty$ then $\underline{H}_{f}^{U, x}$ does not take the value $-\infty$ and

$$
\underline{h}_{f}^{J, U} \geq \underline{h}_{f}^{U}, x+\underline{H}_{f}^{U, x} .
$$

From these considerations we see that if $\bar{h}_{f}^{U, U}$ (resp. $\underline{h}_{f}^{U, v}$ ) is finite then $\bar{H}_{f}^{U, x}$ (resp. $\underline{H}_{f}^{U, x}$ ) is finite and $f$ is therefore resolutive (Theorem 1.1). If $\bar{h}_{f}^{u, x}$ and $\underline{h}_{f}^{U, x}$ are finite then $\bar{H}_{f}^{U, x}$ (resp. $\underline{H}_{f}^{U, x}$ ) does not take the value $+\infty$ (resp. $-\infty)$; these functions are therefore finite and $f$ is resolutive.

Suppose now $f$ resolutive. Let $s \in \overline{\mathscr{W}}_{f}^{U, U}$ and $\underline{s} \in \underline{\mathscr{L}}_{f}^{U, x}$. Then $s-\underline{s} \in \overline{\mathscr{W}}_{f}^{U, x}$ and therefore

$$
s-\underline{s} \geq \bar{h}_{f}^{U}, x
$$

$s$ and $\underline{s}$ being arbitrary we get

$$
\bar{h}_{f}^{U, U} \geq \bar{h}_{f}^{U, x}+H_{f}^{U, x} .
$$

The converse inequality follows from the first part of the proof. The proof of the second equality is similar.

2.2. Let $U$ be an open subset of $X, U \in \mathfrak{P}$ and $f$ be a real function defined outside a compact set of $X$. We say that $f$ is associated with zero at the Alexandroff point of $\boldsymbol{X}$ along $U$ if $\bar{h}_{|f|}^{r} x=0$. If $U \subset V \in \mathfrak{B}$ and $f$ is associated with zero at the Alexandroff point of $X$ along $V$ then it is associated with zero at the Alexandroff point of $X$ along $U$. A potential on $X$ is associated with 
zero at the Alexandroff point of $X$ along $X$. If $X \notin \Re$ then any non-negative superharmonic function $s$ on $X$ is associated with zero at the Alexandroff point of $X$ along any open subset $U$ of $X, U \in \mathfrak{P}$. Indeed let $\bar{s} \in \overline{\mathscr{S}}_{s}^{U, X}$; the function on $X$ equal to $s$ on $X-U$ and equal to $\min (s, \bar{s})$ on $U$ is superharmonic, nonnegative. Since $X \notin \mathfrak{P}$ it must be equal to $s$. We deduce $s=H_{s}^{t s, x}, \bar{h}_{s}^{U,}, x=0$ (Lemma 2.4).

TheOREm 2.1. Let $U$ be an open subset of $X, U \in \mathfrak{P}$, and $s$ be a non-negative superharmonic function on $X$. The following two conditions are equivalent:

a) $s$ is associated with zero at the Alexandroff point of $X$ along $U$;

b) any superharmonic function $s^{\prime}$ on $U$ is non-negative if

$$
\liminf _{x \rightarrow y} s^{\prime}(x) \geq 0
$$

for any $y \in \partial U$ and if there exists a positive number $\alpha$ such that $s^{\prime} \geq-\alpha s$ outside a compact set of $X$.

$a \Rightarrow b$. By Lemma 2.2 there exists a non-negative superharmonic function $s_{0}$ on $U$ such that for any $\varepsilon>0 \varepsilon s_{0} \in \overline{\mathscr{W}}_{s}^{U, x}$. Then $s^{\prime}+\varepsilon s_{0} \in \overline{\mathscr{W}}_{0}^{U, x}$ for any $\varepsilon>0$. Hence $s^{\prime}+\varepsilon s_{0} \geq 0$. $\varepsilon$ being arbitrary, $s^{\prime}$ is non-negative.

$b \Rightarrow a$. We may suppose $U$ connected. Let $p$ be an Evans potential on $U$. Since $\bar{h}_{s}^{(\tau, x}$ is associated with zero at any boundary point of $U$ we see by Lemma 1.6 that $p-\bar{h}_{s}^{v, x}$ satisfies the conditions of $b$ ) with $\alpha=1$. Hence it is nonnegative and $\bar{h}_{s}^{U, x}$ vanishes, being dominated by a potential.

2.3. Let $U$ be an open set on $X, U \in \mathfrak{P}$, and $f$ be a real function defined on $U$ outside a compact set of $X$. The function $f$ is called harmonizable with respect to $(U, X)$ if $\bar{h}_{f}^{U, x}, \underline{h}_{f}^{U, x}$ are finite and equal; in this case we denote by $h_{f}^{U, X}=h_{f}^{U}=h_{f}$ their common value. Instead of harmonizable with respect to $(U, U)$ we shall say harmonizable on $U$.

THEOREM 2.2. Let $U$ be an open subset of $X, U \in \mathfrak{P}$, and $f, f^{\prime}$ be real functions defined on $U$ outside a compact set of $X$, harmonizable with respect to $(U, X)$ and let $\alpha$ be a real number. Then $\alpha f^{*)}, f+f^{(*)}, \max \left(f, f^{\prime}\right), \min \left(f, f^{\prime}\right)$ are also harmonizable with respect to $(U, X)$ and we have

*) Here $a f$ and $f+f^{\prime}$ are defined arbitrary when the operations have no sense. 


$$
\begin{array}{ll}
h_{\alpha f}=\alpha h_{f}, & h_{f+f^{\prime}}=h_{f}+h_{f^{\prime}}, \\
h_{\max \left(f^{\prime}, f^{\prime}\right)}=h_{f} \vee h_{f^{\prime}}, & h_{\min \left(f_{,} f^{\prime}\right)}=h_{f} \wedge h_{f^{\prime}}, \\
h_{f}=0 \Rightarrow h_{\mid f_{1}}=0 . &
\end{array}
$$

This theorem follows immediately from Lemma 2.1 .

Theorem 2.3. Let $U, U^{\prime}$ be open subsets of $X, U \subset U^{\prime}, U^{\prime} \in \mathfrak{P}$, and let $f$ be a real function defined on $U^{\prime}$ outside a compact set of $X$. If $f$ is harmonizable with respect to $\left(U^{\prime}, X\right)$ and if we denote $f^{\prime}=h_{f}^{U^{\prime}}, x, f$ and $f^{\prime}$ are harmonizable with respect to $(U, X)$ and $h_{f}^{U, X}=h_{f^{\prime}}^{U}$.

In order to prove that $f^{\prime}$ is harmonizable with respect to $(U, X)$ we may suppose $f$ non-negative. There exists by Lemma 2.2, a non-negative superharmonic function $s^{\prime}$ on $U^{\prime}$ such that $f^{\prime}-\varepsilon s^{\prime} \in \mathscr{E}_{f}^{U^{\prime}}, x$ for any $\varepsilon>0$. Let $p$ be an Evans potential on $U$. Since $\bar{h}_{f}^{U}{ }^{\prime}{ }^{x}$ is associated with zero at any point of $U^{\prime} \cap$ $\partial U$ and since it is dominated by $f^{\prime}$ on $U, \bar{h}_{f^{\prime}}^{U, x}-p-\varepsilon s^{\prime} \in \underline{\mathscr{L}}_{f^{\prime}}^{U}{ }^{x}$,

$$
\bar{h}_{f}^{U} ; x-p-\varepsilon s^{\prime} \leq \underline{h}_{f}^{U} ; x \text {. }
$$

$p$ and $\varepsilon$ being arbitrary $f^{\prime}$ is harmonizable with respect to $(U, X)$.

The other assertions of the theorem follow now from Lemma 2.5.

Corollary 2.1. Let $\mathcal{S}$ be a set of superharmonic functions on $X, f$ be the greatest lower bound of $\mathcal{S}$ and $U$ be an open subset of $X, U \in \Re$. If $f$ possesses a non-positive subharmonic minorant it is harmonizable with respect to $(U, X)$.

If $X \notin \mathfrak{P}$ then $f$ is associated with zero at the Alexandroff point of $X$ along $U$. If $X \in \mathfrak{P}$, by the preceding theorem it is sufficient to prove that $f$ is harmonizable on $X$. Obviously $\bar{h}_{f} \leq \bar{h}_{s} \leq s$ for any $s \in \mathscr{S}$. Hence $\bar{h}_{f} \in \underline{\mathscr{W}}_{f}, \bar{h}_{f} \leq \underline{h}_{f}$.

Corollary 2.2. Let $X \in \mathfrak{B}, U$ be an open subset of $X$ and $f$ be a real function defined on $X$ outside a compact set such that $f$ vanishes quasi-everywhere on $X-U$ outside a compact set and $|f|$ is dominated by a superharmonic function on $X$. If $f$ is harmonizable on $X$ and $h_{f}^{T, X}=0$ then $h_{f}^{X, X}=0$.

Let us denote $u=h_{|f| X}^{X}$. By the theorem

$$
h_{u}^{U, x}=h_{\mid f}^{U, x}=0 \text {. }
$$

Hence by Lemma 2.7 and Corollary 1.1

$$
u=h_{u}^{U, U}=H_{u}^{U, x}, \quad u=\hat{R}_{u}^{X-\varepsilon} .
$$


From Lemma 2.6 it follows that $u$ is a potential. Hence $u=0$.

Theorem 2.4. Let $U . U^{\prime}$ be open subsets of $X, U \subset U^{\prime}, U \in \mathfrak{P}$, and let $f$ be a real function defined on $U$ outside a compact subset of $U^{\prime}$. If $f$ is harmonizable with respect to $\left(U, U^{\prime}\right)$ and there exists a superharmonic function on $X$ dominating $|f|$ then $f$ is harmonizable with respect to $(U, X)$. If $h_{f}^{U, U^{\prime}}=0$ then $h_{f}^{\pi, x}=0$.

Suppose firstly $f$ harmonic on $U$ and non-negative. Since $f$ is dominated by a superharmonic function on $X, \bar{h}_{f}^{T,}{ }^{X}$ is associated with zero at any point of $\partial U$. Hence for any Evans potential $p$ on $U, \bar{h}_{f}^{(f, x}-p \in \mathscr{Q}_{f}^{(j, x}$ and $f$ is harmonizable with respect to $(U, X)$.

In virtue of Theorem 2.2 and the above considerations we may suppose that $h_{f}^{r, U^{\prime \prime}}=0$. In this case again by Theorem 2.2 we have $h_{\mid}^{l^{\prime}} \dot{f}^{*^{\prime}}=0$. Since $\overline{\mathscr{W}}_{|j|}^{U, U^{\prime}} \subset \overline{\mathscr{W}}_{|j|}^{U}{ }^{X}$ we get $\bar{h}_{|j|}^{U,}{ }^{x}=0 . \quad f$ is therefore harmonizable with respect to $(U, X)$.

Theorem 2.5. Let $X \in \mathfrak{B}, U$ be an open subset of $X$, s be a non-negative superharmonic function on $X$ such that $\hat{R}_{s}^{X-U}$ is a potential and $f$ be a real function defined on $X$ outside a compact set, $|f| \leq s$. If $f$ is harmonizable with respect to $(U, X)$ then $f$ is harmonizable on $X$. If $h_{f}^{T, X}=0$ then $h_{f}^{X, X}=0$.

We denote $u=\bar{h}_{f}^{X}, x-\underline{h}_{f}^{X}, x$ (resp. $u=\bar{h}_{|f|}^{X}, X$ if $h_{f}^{U, x}=0$ ). The functions $\bar{h}_{f}^{X}, x$, $\underline{h}_{f}^{X}, x, \bar{h}_{|f| x \mid}^{X, X}$ are finite, since $|f| \leq s$, and by Lemma 2.5. we have $h_{u}^{h, x}=0$. By Lemma 2.7 and Corollary 1.1 we get

$$
\boldsymbol{u}=h_{u}^{U, U}=H_{u}^{i,}, \quad u=\hat{R}_{u}^{X-U^{t}} \leq 2 \hat{R}_{s}^{X-c^{*}} .
$$

Being dominated by a potential, $u$ vanishes.

COROLlary 2.3. Under the same hypothesis if $f$ is harmonizable on $U$ then $f$ is harmonizable on $X$. If $h_{f}^{U, U}=0$ then $h_{f}^{X, X}=0$.

The assertion follows immediately from the theorem using Theorem 2.4.

Corollary 2.4. Let $X \in \mathfrak{P}, U$ be an open subset of $X$, such that $X-U$ is compact and $f$ be a real function defined on $X$ outside a compact set. If $f$ is harmonizable with respect to $(U, X)$ then $f$ is harmonizable on $X$. If $h_{f}^{T, X}=0$ then $h_{f}^{x}, x=0$.

Let $s$ be a superharmonic function from $\left.\overline{\mathscr{W}}_{|f|}^{i}\right|^{x}$. It can be shown like in [2] (Theorem 14) the existence of a positive superharmonic function $s^{\prime}$ on $X$ 
which dominates $s$ on $U$ outside a compact subset of $X$. Since $\hat{R}_{S^{\prime}}^{X-U}$ is a potential the conditions of the theorem are fulfilled.

Corollary 2.5. Let $X \in \mathfrak{P}, U_{1}, U_{2}$ be open disjoint subsets on $X$ and $f_{1}$, $f_{2}$ be harmonizable functions on $X$. If $X-U_{1} \cup U_{2}$ is compact then the function equal to $f_{1}$ on $U_{1}$ and equal to $f_{2}$ on $U_{2}$ is harmonizable on $X$.

By Theorem 2.3 this function is harmonizable with respect to $\left(U_{i}, X\right) \quad(i=$ $1,2)$ and therefore with respect to $\left(U_{1} \cup U_{2}, X\right)$.

Lemma 2.8. Let $X \in \mathfrak{P}$ and let $f, g$ be real finite funitions on $X, g \geq 0$, and locally bounded such that $g$ and $f g$ are harmonizable on $X$. Let $\alpha, \beta$ be real numbers $\alpha<\beta$ and $A=\{x \in X \mid f(x) \leq \alpha\}, B=\{x \in X \mid f(x) \geq \beta\}$. Then $\min \left(\hat{R}_{g}^{A}, \hat{R}_{g}^{B}\right)$ is a potential.

Denote

$$
\gamma=\frac{\alpha+\beta}{2}, \quad f_{A}=\max \left(\frac{\gamma-f}{\gamma-\alpha}, 0\right) \quad f_{B}=\max \left(\frac{f-\gamma}{\beta-\gamma}, 0\right) .
$$

The functions $g f_{A}, g f_{B}$ are harmonizable. We put $u=h_{g f_{A}}, v=h_{g f_{B}}$. Then

$$
h_{\min (u, v)}=h_{u} \wedge h_{v}=u \wedge v=h_{\min \left(g f_{A}, g f_{B}\right)}=0 .
$$

$\min (u, v)$ is therefore a potential. On the other hand, by Lemma 2.2 there exists a potential $p$ such that

$$
g f_{A} \leq u+p, \quad g f_{B} \leq v+p .
$$

Since $f_{A}$ (resp. $\left.f_{B}\right)$ is not smaller than 1 on $A$ (resp. $B$ ) we have

$$
\begin{gathered}
\hat{R}_{g}^{A} \leq u+p, \quad \hat{R}_{g}^{B} \leq v+p, \\
\min \left(\hat{R}_{g}^{A}, \hat{R}_{g}^{B}\right) \leq \min (u, v)+p,
\end{gathered}
$$

and $\min \left(\hat{R}_{g}^{A}, \hat{R}_{g}^{B}\right)$ is a potential being dominated by a potential.

Theorem 2.6. Let $X \in \mathfrak{P}, X$ connected, $g$ be a non-negative locally bounded harmonizable function on $X, f$ be a real finite function on $X$ and for any real number a denote

$$
A_{\alpha}=\{x \in X \mid f(x)=\alpha\} .
$$

If $f g$ is harmonizable then $\hat{R}_{g}^{A \alpha}$ is a potential except for a countable set of $\alpha$. Conversely if $f$ is continuous and bounded and the set of $\alpha$ for which $\hat{R}_{g}^{A \alpha}$ is a potential is dense then $\mathrm{fg}$ is harmonizable. 
Let us denote for any real number $\alpha s_{x}=R_{g}^{4 \alpha}$. Then $h_{s_{\alpha}}=h_{\hat{s}_{\alpha}} \leq h_{g}$.

Suppose firstly that $f g$ is harmonizable. From the preceding lemma we see that for any two different real numbers $\alpha, \beta$

$$
h_{s_{\alpha}} \wedge h_{s_{\beta}}=h_{\min \left(\hat{s}_{\alpha}, \dot{s}_{\beta}\right)}=0 .
$$

Therefore

$$
\sum_{\alpha} h_{s_{\alpha}}=\underset{\alpha}{\vee} h_{s_{\alpha}} \leq h_{g}
$$

and $h_{s_{\alpha}}$ is different from zero only for a countable set of $\alpha$.

Suppose now that $f$ is continuous and bounded and let $Z$ denote the set of real numbers $\alpha$ for which $\hat{s}_{\alpha}$ is a potential. We may suppose $0<f<1$. Since $\left|g-h_{g}\right|$ is dominated outside a compact set by a potential the function $\hat{R}_{g}^{A \alpha}$ is a potential (resp. the function $f g$ is harmonizable) if and only if $\hat{R}_{h g}^{A \alpha}$ is a potential (resp. $f h_{g}$ is harmonizable) and therefore we may suppose $g$ is harmonic. Let $\varepsilon$ be a positive number and $\left\{\alpha_{i}\right\}_{0 \leq i \leq n}$ numbers from $Z$ such that

$$
\begin{aligned}
& 0=\alpha_{0}<\alpha_{1}<\cdots<\alpha_{n}=1, \\
& \alpha_{i}-\alpha_{i-1}<\varepsilon \\
& F_{i}=\left\{x \in X \mid \alpha_{i-1} \leq f(x) \leq \alpha_{i}\right\}, \\
& G_{i}=\left\{x \in X \mid \alpha_{i-1}<f(x)<\alpha_{i}\right\} .
\end{aligned}
$$

Un $X-F_{i}, r_{i}=R_{g}^{F_{i}}$ is equal to $R_{g}^{A \alpha_{i-1} \cup A_{\alpha_{i}}}$ and therefore smaller than $s_{\alpha_{i-1}}+s_{\alpha_{i}}$. Hence $R_{r_{i}}^{X-F_{i}}$ is a potential. We have

$$
\begin{aligned}
& \sum_{i=1}^{n} \alpha_{i-1}\left(r_{i}-R_{r_{i}}^{X-F_{i}}-s_{\alpha_{i}}\right) \leq f g \leq \sum_{i=1}^{n} \alpha_{i} r_{i}, \\
& \sum_{i=1}^{n}\left(r_{i}-R_{r_{i}}^{X-F_{i}}-s_{\alpha_{i}}\right) \leq g .
\end{aligned}
$$

Since $R_{r_{i}}^{X-F_{i}}$ and $\hat{s}_{\alpha_{i}}$ are potentials we deduce

$$
\begin{aligned}
& \sum_{i=1}^{n} \alpha_{i-1} h_{r_{i}} \leq \underline{h}_{f g} \leq \check{h}_{f g} \leq \sum_{i=1}^{n} \alpha_{i} h_{r_{i}}, \\
& \sum_{i=1}^{n} h_{r_{i}} \leq h_{g}, \quad \bar{h}_{f g}-\underline{h}_{f g} \leq \varepsilon \sum_{i=1}^{n} h_{r_{i}} \leq \varepsilon g .
\end{aligned}
$$

$\varepsilon$ being arbitrary $f g$ is harmonizable.

2.4. Lemma 2.9. Let $f$ be a continuous finite function on $X$ and $U$ an open subset of $X, U \in \mathfrak{P}$.

a) $f$ is harmonizable on $U$ if and only if it is harmonizable with respect to 
$(U, X)$.

b) If $X \in \mathfrak{H}$ and $f$ is harmonizable on $X$ then $f$ is harmonizable on $U$.

c) If $X \in \mathfrak{B}, X-U$ is compact and $f$ is harmonizable on $U$ it is harmonizable on $X$.

a) follows immediately from Lemma 2.7 . b) results from Theorem 2.3 and from a). c) may be obtained using Corollary 2.4 and a).

$A$ continuous finite function $f$ on $X$ is called $a$ Wiener function if there exists an open set $U$ with compact complement, $U \in \mathfrak{P}$, such that $f$ is harmonizable on $U$. From the preceding lemma the last assertion is equivalent to the assertion that $f$ is harmonizable with respect to $(U, X)$.

If $X$ is connnected and belongs to $\mathfrak{g}-\mathfrak{P}$ then any continuous function on $X$ dominated by a superffarmonic function is a Wiener function.

Theorem 2.7. If $f$ is a Wiener function and $U$ an open set, $U \in \mathfrak{P}$, then $f$ is harmonizable on $U$.

By the definition there exists an open set $V$ with compact complement, $V \in \mathfrak{B}$, such that $f$ is harmonizable on $V$. If $U \subset X-V$ then $U$ is relatively compact and $f$ is harmonizable by Lemma 2.7. On the contrary case let $K$ be a compact non-polar subset of $U \cap V$. Then $X-K \in \mathfrak{P}$ by Theorem 7 in [3]. By b) of the preceding lemma $f$ is harmonizable on $V-K$ and by c) it is harmonizable on $X-K$. Again by b) $f$ is harmonizable on $U-K$ and by c) on $U$.

REMARK. The restriction of a Wiener function to an open subset $U$ is a Wiener function on $U$. If $X \in \mathfrak{P}$, a continuous finite function is a Wiener function if and only if it is harmonizable on $X$. We denote by $\mathscr{W}(X)=\mathscr{W}$ the set of all Wiener functions on $X$. From Theorem 2.2 and Theorem 2.7 we see that $\mathscr{W}$ is a real vector space and from $f_{1}, f_{2} \in \mathscr{H}$ it follows $\max \left(f_{1}, f_{2}\right), \min \left(f_{1}, f_{2}\right)$ $\in \mathscr{W}$. If $X$ belongs to $\mathfrak{B}$ we denote by $\mathscr{W}_{0}(X)=\mathscr{W}_{0}$ the set of Wiener functions $f$ for which $h_{f}^{X}$ vanishes and call the functions of $\mathscr{W}_{0}$ Wiener potentials. $\mathscr{W}_{0}$ coincides with the set of continuous finite functions whose moduli are dominated by a potential, (Lemma 2.2).

LEMMA 2.10. If $X \in(\mathfrak{2}-\mathfrak{P}) \cup(\mathfrak{B}-\mathfrak{S})$ then any real finite continuous function $f$ on $X$ such that $|f|$ possesses a superharmonic majorant is a Wiener function.

Let $s$ be a superharmonic majorant of $|f|$. Since for any $U \in \mathfrak{F}, s$ is as- 
sociated with zero at the Alexandroff point of $X$ along $U$ we have

$$
0 \leq \bar{h}_{|f|}^{U, x} \leq h_{s}^{U, x}=0
$$

and $f$ is harmonizable with respect to $(U, X)$.

Lемма 2.11. Let $f$ be $a$ Wiener function on $X$ and $U$ be an open subset of $X, U \in \mathfrak{R}$. If the function $g$ on $X$ equal to $f$ on $X-U$ and equal to $H_{f}^{J,}{ }^{x}$ on $U$ is continuons then it is a Wiener function.

Suppose firstly $X \in \mathfrak{B}$ and let $f^{\prime}$ be a locally bounded harmonizable function on $X$ such that $\bar{H}_{f}^{U} ;{ }^{X}$ is finite and $g^{\prime}$ be the function on $\mathrm{X}$ equal to $f^{\prime}$ on $X-U$ and equal to $\bar{H}_{f ;}^{f^{\prime} x}$ on $U$. Denote $u=h_{f}$. There exists a potential $p$ dominating $\left|f^{\prime}-u\right|$. Then $p$ dominates $\left|g^{\prime}-R_{u v_{0}}^{X-U}+R_{(-u) v_{0}}^{X-U}\right|$ and $g^{\prime}-R_{u v_{0}}^{X-U}+R_{(-u) \vee_{0}}^{X-U}$ is harmonizable. Since $R_{u \vee 0}^{X-U}, R_{(-\boldsymbol{u}) \vee_{0}}^{X-U}$ are harmonizable (Corollary 2.1), $g^{\prime}$ is harmonizable.

Suppose now $X \notin \Re$ and let $K$ be a non-polar compact subset of $X-U$. Then $X-K \in \mathfrak{B}$. We denote by $g_{0}$ the function on $X-K$ equal to 0 on $(X-K)$ - $U$ and equal to $H_{\psi}^{U, x}$ on $U$, where $\psi$ is equal to $f$ on $K \cap \partial U$ and equal to 0 on $\partial U-K$. Since $|f|$ possesses a superharmonic majorant on $X-K, H_{\psi}^{U, x}$ is associated with zero at any point of $\partial U-K$. Hence by Theorem 2.2, Theorem 1.2 and Corollary 2.1, $g_{0}$ is harmonizable on $X-K$. We set $f^{\prime}=f-g_{0}$ and denote by $g^{\prime}$ the function on $X-K$ equal to $f^{\prime}$ on $(X-K)-U$ and equal to $H_{f^{\prime}}^{\pi, X-K}$ on $U$. Since $f^{\prime}$ is harmonizable on $X-K, g^{\prime}$ is harmonizable on $X-K$ by the above proof. From the equality $g=g^{\prime}+g_{0}$ it follows that $g$ is harmonizable on $X-K . \quad g$ is therefore a Wiener function.

Lемма 2.12. Let $X$ be non-compact, $X \in \mathfrak{P}$, and $f$ be a real function on $X$, whose modulus possesses a superharmonic majorant and such that

$$
\lim \sup \sigma^{*} \underline{H}_{f}^{U} \leq 0^{*)}
$$

Let further $g$ be a non-negative harmonizable function on $X$ and $F$ be a closed set such that $g \leq f$ on $F$. Then $\hat{R}_{g}^{F}$ is a potential.

Let us denote $u=h_{g}$. There exists a potential $p$ dominating $|u-g|$ outside a compact set. It is sufficient to prove that $\hat{R}_{*}^{F}$ is a potential. Let us denote $f^{\prime}=u-f$ and $G=X-F$. Since $|f|$ possesses a superharmonic majorant there

*) (5) is the filter defined in the page 8 . 
exists a superharmonic majorant $s$ for $\left|f^{\prime}\right|$.

Let $U \in \mathbb{B}, \bar{s} \in \overline{\mathscr{W}}_{s}^{G, x}, \underline{s} \in \underline{\mathscr{P}}_{s}^{G \cup U, x}$. The function $R_{s}^{\prime \prime}+\bar{s}-\underline{s}$ is hyperharmonic on $G$, non-negative on $U \cap G$ and outside a compact subset of $X$ and its lower limit at any point of $\partial G-U$ is non-negative. Hence this function is non-negative. $\bar{s}, \underline{s}$ being arbitrary we get

$$
h_{s}^{G \cup U, x} \leq h_{s}^{G, x}+R_{s}^{U}
$$

on $G$. If we denote for any $U \in \mathbb{B}$ by $s_{U}$ the function on $X$, equal to 0 on $X-G \cup U$ and equal to $h_{s}^{G \cup U, x}$ on $G \cup U$ we get from the above inequality

$$
\begin{aligned}
& s_{U} \leq s_{\phi}+R_{s}^{r}, \\
& s_{U} \leq h_{s_{U}}^{X} \leq h_{s_{\phi}}^{X},
\end{aligned}
$$

since $R_{s}^{U}$ is a potential and $s_{\sigma}$ is nearly subharmonic.

We have for $U, U^{\prime} \in \mathbb{B}, U \subset U^{\prime}, U$ sufficiently large,

$$
\bar{H}_{f^{\prime}}^{U \cup\left(G \cap U^{\prime}\right)} \leq\left(\hat{R}_{s}^{G-U^{\prime}}\right)_{Q \cup E}+p .
$$

Since by Lemma 2.3

$$
s_{U}=h_{s}^{G \cup U, x}=\lim _{U^{\prime}, \Phi^{*}}\left(\hat{R}_{s}^{G-U^{\prime}}\right)_{Q \cup U}
$$

we get

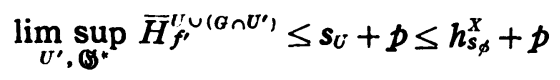

on $G \cup U$. Since

$$
\bar{H}_{f^{\prime}}^{U\left(a \cap U^{\prime}\right)}=u-\underline{H}_{f}^{U U\left(a \cap U^{\prime}\right)}
$$

we have

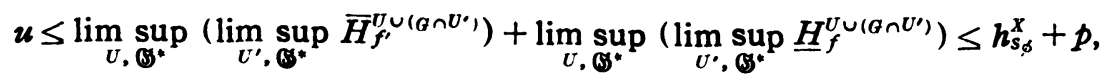

$$
\begin{aligned}
& \hat{R}_{u}^{p} \leq \hat{R}_{v}^{p}+p,
\end{aligned}
$$

where $v$ denotes $h_{s_{\phi}}^{X}$. Since by Lemma 2.6, $\hat{R}_{v}^{F}$ is a potential, $\hat{R}_{u}^{p}$ is a potential.

THEOREM 2.8. Let $X$ be non-compact, $X \in \mathfrak{B}$, and $f$ be a finite continuous function on $X$ whose modulus is dominated by a superharmonic function. If

$$
\lim \boldsymbol{s}^{*} H_{f}^{v}
$$

exists then $f$ is a Wiener function and

$$
\lim _{\sigma^{*}} H_{f}^{u}=h_{f}^{X} .
$$

Let $s$ be a continuous finite superharmonic majorant of $|f|$. 
Suppose firstly

$$
\lim _{\mathscr{S}^{*}} H_{f}^{v}=0 .
$$

Let $\varepsilon$ be a positive number and

$$
F=\{x \in X \mid \operatorname{es}(x) \leq f(x)\} .
$$

Let $s^{\prime}$ be a non-negative hyperharmonic function dominating $s$ on $F$. Then $s^{\prime}+\varepsilon s \in \overline{\mathscr{W}}_{f}$. Hence

$$
\bar{h}_{f} \leq \boldsymbol{s}^{\prime}+\varepsilon s .
$$

$s^{\prime}$ being arbitrary we get $\bar{h}_{f} \leq \hat{R}_{s}^{F}+\varepsilon s$ and therefore $\bar{h}_{f} \leq \varepsilon s$ since by the preceding lemma $\hat{R}_{s}^{F}$ is a potential. Similarly $\underline{h}_{f} \geq-\varepsilon s$. Hence $h_{f}=0$.

For the general case let us denote

$$
u=\lim _{\mathbb{S}^{*}} H_{f}^{c^{\prime}}
$$

We want to prove firstly that $u$ is harmonic. We may assume $X$ is connected. Let $\left\{U_{n}\right\}$ be a pseudo-exhaustion of $X, p$ be a potential on $X$ equal to infinite on $X-\bigcup_{n=1}^{\infty} U_{n}$ and finite on $\bigcup_{n=1}^{\infty} U_{n}, x$ be a point of $\bigcup_{n=1}^{\infty} U_{n}$ and $\varepsilon$ be a positive number. There exists a relatively compact open set $U$ of $X, x \in U$, such that

$$
\left|H_{f}^{U^{\prime}}(\boldsymbol{x})-\boldsymbol{u}(\boldsymbol{x})\right|<\varepsilon
$$

for any relatively compact open set $U^{\prime} \supset U$. Let $\alpha$ be a positive number and

$$
G=\{y \in X \mid p(y)>\alpha s(y)\} .
$$

$\bar{U}-G$ is a compact subset of $\bigcup_{n=1}^{\infty} U_{n}$ and we have therefore, for a sufficiently large $n, \bar{U}-G \subset U_{n}$. Let us denote by $g$ the function on $\overline{U \cup U_{n}}$ equal to $f$ on $\partial\left(U \cup U_{n}\right)$ and equal to $H_{f}^{I V U_{n}}$ on $U \cup U_{n}$. Obviously $|g| \leq s$. By Lemma 1.2

$$
H_{f}^{U \cup U_{n}}=H_{g}^{U_{n}} .
$$

Since $|f-g|$ is equal to zero on $\partial U_{n}-G$ and dominated by $\frac{2 p}{\alpha}$ on $G \cap \partial U_{n}$ we get

$$
\left|H_{f}^{U_{n}}-H_{g}^{U_{n}}\right| \leq H_{|f-g|}^{U_{n}} \leq \frac{2 p}{\alpha}
$$

Hence

$$
\begin{aligned}
& \left|H_{f}^{U n}(x)-u(x)\right| \leq\left|H_{f}^{U, n}(x)-H_{g}^{U n}(x)\right|+\left|H_{f}^{U U_{n}}(x)-u(x)\right| \leq \varepsilon+\frac{2 p(x)}{\alpha} . \\
& \underset{n \rightarrow \infty}{\lim _{n \rightarrow \infty} \sup ^{\prime \prime}}\left|H_{f}^{\prime \prime n}(x)-u(x)\right| \leq \varepsilon+\frac{2 p(x)}{\alpha}
\end{aligned}
$$


$\varepsilon$ and $\alpha$ being arbitrary we get

$$
\lim _{n \rightarrow \infty} H_{f}^{U_{n}}(x)=u(x)
$$

Let $V$ be an arbitrary regular domain, $x \in V$, and $\left\{U_{n}\right\}$ be a pseudo-exhaustion of $X$ such that $\bar{V} \subset U_{1}$. We put $u_{n}=H_{f}^{I t n}$. Then $\left|u_{n}\right|<s$ and

$$
u(x)=\lim _{n \rightarrow \infty} u_{n}(x)=\lim _{n \rightarrow \infty} \int u_{n} d \omega_{x}=\int u d \omega_{x}
$$

$V$ and $x$ being arbitrary $u$ is harmonic.

We have

$$
\lim _{\mathscr{S}^{*}} H_{f-\boldsymbol{u}}^{U}=0 .
$$

By the first part of the proof we see that $f-u$ is a Wiener potential. Since $|u|$ possesses a superharmonic majorant, $u$ is a Wiener function. Hence $f$ is a Wiener function and we have

$$
h_{f}^{X}=h_{f-u}^{X}+h_{u}^{X}=u .
$$

\section{§ 3. Harmonic maps}

3.1. Let $X, X^{\prime}$ be two harmonic spaces. A continuous map $\varphi: X \rightarrow X^{\prime}$ is called a harmonic map if for any open set $U^{\prime} \subset X^{\prime}$ and any harmonic function $u^{\prime}$ on $U^{\prime}, u^{\prime} \circ \varphi$ is harmonic on $\varphi^{-1}\left(U^{\prime}\right)$. The composition of two harmonic maps and the canonic injection of an open set of $X$ in $X$ are harmonic maps. If $\varphi: X \rightarrow X^{\prime}$ is a harmonic map and $f^{\prime}$ is a positive continuous finite function on $X^{\prime}$ and if we denote by $Y$ (resp. $Y^{\prime}$ ) the harmonic space obtained from $X$ (resp. $X^{\prime}$ ) dividing the sheaf of harmonic functions by $f^{\prime} \circ \varphi\left(\right.$ resp. $\left.f^{\prime}\right)$ then $\varphi: Y \rightarrow Y^{\prime}$ is also a harmonic map.

TheOREM 3.1. If $\varphi: X \rightarrow X^{\prime}$ is a harmonic map and $s^{\prime}$ a hyperharmonic function on an open set $U^{\prime} \subset X^{\prime}$, then $s^{\prime} \circ \varphi$ is a hyperharmonic function on $\varphi^{-1}\left(U^{\prime}\right)$.

We may suppose $s^{\prime}$ is superhamonic. Let $V$ be a relatively compact open set, $\bar{V} \subset \varphi^{-1}\left(U^{\prime}\right)$. Then $\varphi(\bar{V})$ is a compact subset of $U^{\prime}$. Let $\mathfrak{B}^{\prime}$ be a finite covering of $\varphi(\bar{V})$ with regular domains. We denote by $s_{\mathfrak{B}^{\prime}}^{\prime}$ the function on $U^{\prime}$ equal to $s^{\prime}$ on $U^{\prime}-\bigcup_{V^{\prime} \in \mathfrak{B}^{\prime}} V^{\prime}$ and equal to

$$
\min _{\boldsymbol{x}^{\prime} \in V^{\prime} \in \mathfrak{O}^{\prime}} H_{s^{\prime}}^{\nu^{\prime}}\left(\boldsymbol{x}^{\prime}\right)
$$


at any point $x^{\prime} \in \bigcup_{V^{\prime} \in \mathfrak{Q}^{\prime}} V^{\prime}$. Let $x \in V$ and $U$ be a regular domain, $x \in U \subset V$ such that $\bar{U}$ is contained in any set $\varphi^{-1}\left(V^{\prime}\right)$, for which $\varphi(x) \in V^{\prime} \in \mathfrak{B}^{\prime}$. We denote by $s$

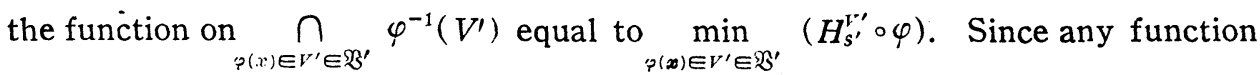
$H_{s^{\prime}}^{r^{\prime \prime}} \circ \varphi$ is harmonic on this set, $s$ is superharmonic. We have $s \geq s_{\mathfrak{B}^{\prime}}^{\prime} \circ \varphi$ and $s(x)=s_{\mathfrak{B}}^{\prime} \circ \varphi(x)$. Hence

$$
s_{\mathfrak{Y}^{\prime}}^{\prime} \circ \varphi(x)=s(x) \geq H_{s}^{U}(x) \geq H_{s^{\prime}}^{U}{ }_{\mathfrak{B}^{\prime \prime \rho}}(x) .
$$

Since $s_{\mathfrak{X}^{\prime}}^{\prime} \circ \varphi$ is continuous it is superharmonic on $V$ (See Theorem 4 in [2]). Obviously $s^{\prime}$ is the least upper bound of the family $\left\{s_{\mathfrak{B}^{\prime}}^{\prime}\right\}_{\mathfrak{B}^{\prime}}$. Since it is upper directed it follows that $s^{\prime} \circ \varphi$ is hyperharmonic.

Corollary 3.1. Let $\varphi: X \rightarrow X^{\prime}$ be a non constant harmonic map, $X$ connected. If $X^{\prime} \in \mathfrak{P}($ resp. Sू), then $X \in \mathfrak{P}$ (resp. Sু).

If $X^{\prime} \in \mathfrak{S}$ there exists a positive harmonic function $\boldsymbol{u}^{\prime}$ on $X$. Then $\boldsymbol{u}^{\prime} \circ \varphi$ is a positive harmonic function on $X$ and $X \in \mathscr{S}$.

Suppose now $X^{\prime} \in \mathfrak{P}$ and let $x_{1}^{\prime}, x_{2}^{\prime}$ be two different points of $\varphi(X)$. There exists two positive finite superharmonic functions $s_{1}^{\prime}, s_{2}^{\prime}$ such that

$$
s_{1}^{\prime}\left(x_{1}^{\prime}\right)=s_{2}^{\prime}\left(x_{1}^{\prime}\right), \quad s_{1}^{\prime}\left(x_{2}^{\prime}\right) \neq s_{2}^{\prime}\left(x_{2}^{\prime}\right) .
$$

Then $s_{1}^{\prime} \circ \varphi, s_{2}^{\prime} \circ \varphi$ are two positive non-proportional superharmonic functions on $X$. Hence $X \in \mathfrak{B}$.

TheOREM 3.2. Let $\varphi: X \rightarrow X^{\prime}$ be a non-constant harmonic map and $X$ be connected. Then for any polar set ${ }^{* \prime} A^{\prime} \subset X^{\prime}, \varphi^{-1}\left(A^{\prime}\right)$ is polar.

Let $A$ denote the set of points $x \in X$ such that for any neighbourhood $U$ of $x, U \cap \varphi^{-1}\left(A^{\prime}\right)$ is non-polar. $A$ is obviously closed. Let $x \in A$, and $s^{\prime}$ be a positive superharmonic function on a neighbourhood $U^{\prime}$ of $\varphi(x)$ infinite on $U^{\prime} \cap A^{\prime}$. Let $y^{\prime} \in U^{\prime}, y^{\prime} \neq \varphi(x)$ and $F^{\prime}, F_{0}^{\prime}$ be two closed sets on $U^{\prime}, U^{\prime}=F^{\prime} \cup F_{0}^{\prime}$, $\varphi(x) \notin F^{\prime}, y^{\prime} \notin F_{0}^{\prime}$. Then

$$
s^{\prime} \leq\left(\hat{R}_{s^{\prime}}^{F)^{\prime}}\right)_{U^{\prime}}+\left(\hat{R}_{s^{\prime}}^{F_{\prime^{\prime}}}\right)_{U^{\prime}} .
$$

Let $U$ be the component of $\varphi^{-1}\left(U^{\prime}\right)$ containing $x$. We have

$$
s^{\prime} \circ \varphi \leq\left(\hat{R}_{S^{\prime}}^{F^{\prime}}\right)_{U} \circ \varphi+\left(\hat{R}_{S^{\prime}}^{r 0^{\prime}}\right)_{U}, \circ \varphi
$$

*) A set $A=X$ is called polar if for any point $x \in X$ there exists a positive superharmonic function on a neighbourhood $U$ of $x$ infinite on $A \cap U$. 
on $U$. Since $s^{\prime} \circ \varphi$ is infinite on the non-polar set $U \cap \varphi^{-1}\left(A^{\prime}\right)$ it is identically infinite. The function $\left(\hat{R}_{S^{\prime}}^{F^{\prime}}\right)_{U} \circ \varphi$ being finite at $x$ it follows that $\left(\hat{R}_{S^{\prime}}^{F_{0^{\prime}}}\right)_{U^{\prime}} \circ \varphi$ is identically infinite. Hence $y^{\prime} \notin \varphi(U) . y^{\prime}$ being arbitrary we get $\varphi(U)=\{\varphi(x)\}$, $\varphi(x) \in A^{\prime}, U \subset A$. Hence $A$ is open and $\varphi$ is locally constant on $A$. Since $X$ is connected either $A$ is empty or $A$ is equal to $X$ and $\varphi$ is constant. By hypothesis $\varphi$ is non-constant. Hence $A$ is empty and $\varphi^{-1}\left(A^{\prime}\right)$ polar.

Corollary 3.2. If $\varphi: X \rightarrow X^{\prime}$ is a non-constant harmonic map, $X$ is connected and $s^{\prime}$ is a superharmonic function on an open set $U^{\prime} \subset X^{\prime}$ then $s^{\prime} \circ \varphi$ is superharmonic on $\varphi^{-1}\left(U^{\prime}\right)$.

Lemma 3.1. Let $\varphi: X \rightarrow X^{\prime}$ be a harmonic map and $x^{\prime} \in X^{\prime}$. If $U$ is an open set on $X$ containing a compact component of $\varphi^{-1}\left(x^{\prime}\right)$ then $x^{\prime}$ is an interior point of $\varphi(U)$.

We may suppose $U$ relatively compact and $\varphi^{-1}\left(x^{\prime}\right) \cap \partial U=\phi$. Then $x^{\prime} \notin \varphi(\partial U)$ and there exists a regular domain $V^{\prime}$ such that $x^{\prime} \in V^{\prime}$ and $V^{\prime} \cap \varphi(\partial U)=\phi$. Denote $V=U \cap \varphi^{-1}\left(V^{\prime}\right)$. The set $V^{\prime}-\varphi(V)$ is open since it is equal to $V^{\prime}-\varphi(\bar{V})$. If it is non-empty we may take a compact non-polar set $K^{\prime}$ contained in it. Then the function $s^{\prime}=\left(\hat{R}_{1}^{K^{\prime}}\right)_{V^{\prime}}$ is harmonic and positive on $V^{\prime}-K^{\prime}$ and converges to zero at any point of $\partial V^{\prime}$. The function $s^{\prime} \circ \varphi$ is harmonic and positive on $V$ and converges to zero at any point of $\partial V$. This is a contradiction and we deduce $\varphi(V)=V^{\prime}$ and the proof is complete.

THEOREM 3.3. A zero-dimensional harmonic map ${ }^{* 1}$ is an open map.

THEOREM 3.4. Let $\varphi: X \rightarrow X^{\prime}$ be a bijective harmonic mäp. Then $\varphi^{-1}$ is also a harmonic map.

From the preceding theorem it results that $\varphi^{-1}$ is continuous. Let $U$ be an open set on $X$ and $u$ be a harmonic function on $U$. We want to prove that $u \circ \varphi^{-1}$ is harmonic on $\varphi(U)$. Let $V^{\prime}$ be a regular domain in $Y^{\prime}, \bar{V}^{\prime} \subset \varphi(U)$, and

$$
v^{\prime}=H_{u \supset \varphi^{-1}}^{r^{\prime}}
$$

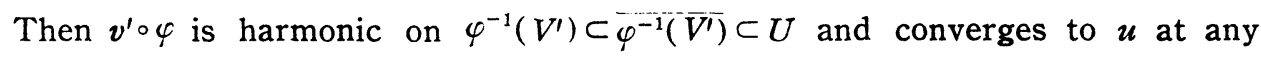
boundary point of $\varphi^{-1}\left(V^{\prime}\right)$. Hence

*) A continuous map $\varphi$ is called zero-dimensional if $\varphi\left(x^{\prime}\right)$ is totally disconnected for any $x^{\prime}$. 


$$
v^{\prime} \circ \varphi=u, \quad v^{\prime}=u \circ \varphi^{-1}
$$

and $u \circ \varphi^{-1}$ is harmonic.

Corollary 3.3. Let $\mathscr{H}$, c岁' be two harmonic sheaves on the same space. If $\mathscr{H}^{\prime} \subset \mathscr{H}$, then $\mathscr{H}^{\prime}=\mathscr{H}$.

Theorem 3.5. Let $\varphi: X \rightarrow X^{\prime}$ be a non-constant harmonic map, $X$ connected. If any point of $X^{\prime}$ is polar, then $\varphi$ is an open map with respect to the fine topologies of $X$ and $X^{\prime}$.

Since the theorem has a local character we may suppose that there exists a positive harmonic function $u^{\prime}$ on $X^{\prime}$. Dividing the sheaf of harmonic functions on $X$ (resp. $\left.X^{\prime}\right)$ with $u^{\prime} \circ \varphi\left(\right.$ resp. $\left.u^{\prime}\right)$ we may suppose further that the constants are harmonic on both spaces.

Let $x \in X$ and $E$ be a fine neighbourhood of $x$. We must prove that $\varphi(E)$ is a fine neighbourhood of $\varphi(x)$. There exists a positive superharmonic function, defined on a neighbourhood of $x$, such that

$$
s(x)<1<\lim _{x \notin y \rightarrow x} \inf _{y \rightarrow \infty}(y) .
$$

Let $U$ be a regular domain containing $x$ such that $s$ is defined on $\bar{U}$ and $s>1$ on $\bar{U}-E$. We denote

$$
G=\{y \in \bar{U} \mid s(y)>1\} .
$$

Obviously $\bar{U}-G \subset E$. Since $\{\varphi(x)\}$ is a polar set $\varphi^{-1}(\varphi(x))$ is also polar (Theorem 3.2). $\varphi^{-1}(\varphi(x)) \cap \partial U$ is therefore of harmonic measure zero with respect to $U$ (See p. 125 of [2]). Let $K$ be a compact subset of $\partial U-\varphi^{-1}(\varphi(x))$ such that

$$
H_{f}^{T}(x)+s(x)<1,
$$

where $f$ denotes the characteristic function of $\partial U-K$. Since $\varphi(x) \notin \varphi(K)$ there exists a regular domain $U^{\prime}, \varphi(x) \in U^{\prime}, \bar{U}^{\prime} \cap \varphi(K)=\phi$. We denote $V=U \cap \varphi^{-1}\left(U^{\prime}\right)$, $G^{\prime}=U^{\prime}-\varphi(\bar{V}-G)$. From

$$
U^{\prime}-G^{\prime} \subset \varphi(\bar{V}-G) \subset \varphi(E)
$$

we see that it is sufficient to prove that $G^{\prime}$ is thin at $\varphi(x)$.

Let $K^{\prime}$ be a compact subset of $G^{\prime}$ and $s^{\prime}=\left(\hat{R}_{1}^{K^{\prime}}\right)_{U^{\prime}} . \quad s^{\prime} \circ \varphi$ is harmonic on $V-\varphi^{-1}\left(K^{\prime}\right)$. The function $H_{f}^{U}+s-s^{\prime} \circ \varphi$ is superharmonic on $V-\varphi^{-1}\left(K^{\prime}\right)$ and its lower limit at any boundary point of $V-\varphi^{-1}\left(K^{\prime}\right)$ is non-negative. Hence it 
is non-negative and we get

$$
\begin{gathered}
\left(\hat{R}_{1}^{K^{\prime}}\right)_{U}(\varphi(x)) \leq H_{f}^{U}(x)+s(x), \\
\left(\hat{R}_{1}^{G^{\prime}}\right)_{U^{\prime}}(\varphi(x))=\sup _{K^{\prime} \subset G^{\prime}}\left(\hat{R}_{1}^{K^{\prime}}\right)_{U}(\varphi(x)) \leq H_{f}^{U}(x)+s(x)<1 .
\end{gathered}
$$

This relation indicates that $G^{\prime}$ is thin at $\varphi(x)$.

Theorem 3.6. Let $\varphi: X \rightarrow X^{\prime}$ be a harmonic map, $X \in \mathfrak{P} \cup \mathfrak{S}, X$ connected. If $U^{\prime}$ is a non-relatively compact domain of $X^{\prime}$ then the set of components of $\varphi^{-1}\left(U^{\prime}\right)$ is at most countable.

Since any component of $\varphi^{-1}\left(U^{\prime}\right)$ is not relatively compact the assertion follows immediately from Theorem 11 in [3].

3.2. Theorem 3.7. Let $\varphi: X \rightarrow X^{\prime}$ be a harmonic map, $X^{\prime} \in \mathfrak{B}$. If $f^{\prime}$ is a Wiener function on $X^{\prime}$, then $f^{\prime} \circ \varphi$ is a Wiener function on $X$.

We may suppose $X$ is connected.

Suppose firstly that $f^{\prime}$ has a compact carrier $K^{\prime}$. Let $s^{\prime}$ be a positive finite superharmonic function on $X^{\prime}$ and $\varepsilon$ be a positive number. There exists two finite potentials $p^{\prime}, p^{\prime \prime}$ on $X^{\prime}$ such that $\left|f^{\prime}-\left(p^{\prime}-p^{\prime \prime}\right)\right|<\varepsilon s^{\prime}$ on $K^{\prime}$ (Theorem 15 in [2]). Then $\left|f^{\prime}-\left(\hat{R}_{p^{\prime}}^{K^{\prime}}-\hat{R}_{p^{\prime}}^{K^{\prime}}\right)\right|<\varepsilon s^{\prime}$ on the whole space $X^{\prime}$ and therefore

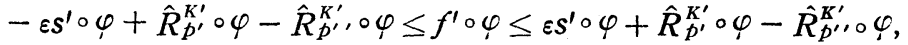

$$
\begin{aligned}
& \bar{h}_{f \prime \circ, p}-\underline{h}_{f \prime \circ p} \leq 2 s s^{\prime} \circ \varphi \text {. }
\end{aligned}
$$

$\varepsilon$ being arbitrary $f^{\prime} \circ \varphi$ is a Wiener function.

Let now $f^{\prime}$ be a Wiener potential. There exists a potential $p^{\prime}$ on $X^{\prime}$ such that for any $\varepsilon>0\left|f^{\prime}\right|<\varepsilon p^{\prime}$ outside a compact set $K_{\varepsilon}^{\prime}$. There exists a continuous finite function $f_{\varepsilon}^{\prime}$ with compact carrier such that $\left|f^{\prime}-f_{\varepsilon}^{\prime}\right|<_{\varepsilon} p^{\prime}$. Hence

$$
\bar{h}_{f \circ \circ,}-\underline{h}_{f \circ \circ p} \leq 2 \varepsilon p^{\prime} \circ \varphi \text {. }
$$

$\varepsilon$ being arbitrary $f^{\prime} \circ \varphi$ is a Wiener function.

If $f^{\prime}$ is a Wiener function the $f^{\prime}-h_{f}$ is a Wiener potential. From

$$
f^{\prime} \circ \varphi=\left(f^{\prime}-h_{f^{\prime}}\right) \circ \varphi+h_{f} \circ \varphi
$$

we see that $f^{\prime} \circ \varphi$ is a Wiener function.

Theorem 3.8. Let $\varphi: X \rightarrow X^{\prime}$ be a harmonic map, $X, X^{\prime}$ connected, $X \in \mathfrak{P}$, $X^{\prime} \in \mathfrak{S}-\mathfrak{P}$. The following assertions are equivalent: 
a) there exists a continuous non-negative non-harmonic function $f^{\prime}$ on $X^{\prime}$ such that $f^{\prime} \circ \varphi$ is a Wiener function on $X$;

b) there exists a closed non-polar set $F^{\prime}$ on $X^{\prime}$ such that $\hat{R}_{1}^{p^{-1}\left(\Gamma^{\prime \prime}\right)}$ is a potential;

c) if $f^{\prime}$ is a locally bounded non-negative function on $X^{\prime}$ such that $f^{\prime} \circ \varphi$ is harmonizable on $X$ and $g^{\prime}$ is a Wiener function on $X^{\prime},\left|g^{\prime}\right| \leq f^{\prime}$, then $g^{\prime} \circ \varphi$ is a Wiener function on $X$.

$a \Rightarrow b$. Let $u^{\prime}$ be a positive harmonic function on $X^{\prime}, g^{\prime}=\frac{f^{\prime}}{u^{\prime}}$ and for any real number $\alpha$

$$
F_{\alpha}^{\prime}=\left\{x^{\prime} \in X^{\prime} \mid g^{\prime}\left(x^{\prime}\right)=\alpha\right\} .
$$

$g^{\prime}$ is continuous and non-constant and therefore for any $\alpha$,

$$
\text { inf } g^{\prime}<\alpha<\sup g^{\prime}
$$

the set $F_{\alpha}^{\prime}$ is non-polar because it separates $X^{\prime}$. The function $\boldsymbol{u}^{\prime} \circ \varphi$ and $\left(g^{\prime} \circ \varphi\right)\left(u^{\prime} \circ \varphi\right)$ are harmonizable. By Theorem 2.6. there exists an $\alpha$,

$$
\inf g^{\prime}<\alpha<\sup g^{\prime}
$$

such that $\hat{R}_{u^{\prime} \circ \rho_{\alpha}^{\prime}}^{p^{-1}\left(F_{\alpha}^{\prime}\right)}$ is a potential. Let $F^{\prime}$ be a compact non-polar subset of $F_{\alpha}^{\prime}$ and

$$
\beta=\inf _{\boldsymbol{x}^{\prime} \in F^{\prime}} u^{\prime}\left(\boldsymbol{x}^{\prime}\right)>0
$$

From

$$
\beta \hat{R}_{i}^{\rho^{-1}\left(F^{\prime \prime}\right)} \leq \hat{R}_{u^{\prime} \% \%}^{-1}\left(F^{\prime \prime} \alpha\right)
$$

we see that $\hat{R}_{1}^{p^{-1}\left(F^{\prime \prime}\right)}$ is a potential.

$b \Rightarrow c$. Let $K^{\prime}$ be a compact non-polar subset of $F^{\prime}$ and

$$
\beta=\sup _{x^{\prime} \in K^{\prime}} f^{\prime}\left(x^{\prime}\right) .
$$

By Lemma 2.2 there exists a potential $p$ on $X$ such that $\left|h_{f \circ \circ p}-f^{\prime} \circ \varphi\right| \leq p$ outside a compact subset $K$ of $X$. Then $s=h_{f^{\prime} \circ p}+p+\hat{R}_{f^{\prime} \circ \%}^{k}$ is a superharmonic majorant of $f^{\prime} \circ \varphi$ on $X$ and $\hat{R}_{s}^{p^{-1}\left(K^{\prime}\right)}$ is a potential being dominated by the potential $\beta \hat{R}_{1}^{\prime{ }^{-1}\left(F^{\prime}\right)}+2 \hat{R}_{s}^{K}+2 p$. By the preceding theorem $g^{\prime} \circ \varphi$ is a Wiener function and therefore harmonizable on $X-\varphi^{-1}\left(K^{\prime}\right), g^{\prime} \circ \varphi$ is therefore a Wiener function on $X$ by Corollary 2.3 .

$c \Rightarrow a$ is trivial since any non-negative continuous function on $X^{\prime}$ dominated by a harmonic function is a Wiener function. 
ThEOREM 3.9. Let $\varphi: X \rightarrow X^{\prime}$ be a harmonic map, $X, X^{\prime}$ connected, $X \in$ $\mathfrak{g}-\mathfrak{B}, X^{\prime} \in \mathfrak{S}-\mathfrak{P}$, and $f^{\prime}$ be a continuous non-negative function on $X^{\prime}$. If $f^{\prime} \circ \varphi$ is a Wiener function on $X$ then for any Wiener function $g^{\prime}$ on $X^{\prime},\left|g^{\prime}\right| \leq f^{\prime}, g^{\prime} \circ \varphi$ is a Wiener function on $X$.

If $f^{\prime}$ is harmonic then it is immediate that $g^{\prime} \circ \varphi$ is a Wiener function. Suppose now $f^{\prime}$ is not harmonic. Let $U$ be a domain on $X, U \in \mathfrak{P}$. We deduce by the preceding theorem $(a \Rightarrow c)$ that $g^{\prime} \circ \varphi$ is a Wiener function on $U$.

3.3. Let $X \in \mathfrak{P} \cup \mathfrak{S}$. We denote by $\Re(X)=\Re$ the set of open subsets $U$ of $X$ such that either $U=\phi$ or $U \in \mathfrak{P}$ and $\bar{h}_{1}^{U, X}=0$. If $U \neq \phi$ this is equivalent with the assertion that 1 is associated with zero at the Alexandroff point of $X$ along $U$. If $V \subset U$ and $U \in \Re$ then $V \in \Re$. Any relatively compact open set

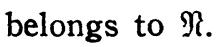

The notion of analytic map of type- $\mathrm{Bl}$ introduced and studied by $\mathrm{M}$. Heins (On the Lindelöf principle. Ann. of Math. 61 (1955), 440-473) can be generalized directly to the case of harmonic maps between spaces belonging to $\mathfrak{F} \cup \mathfrak{S}$. We shall say that a harmonic map $\varphi: X \rightarrow X^{\prime}\left(X, X^{\prime} \in \mathfrak{P} \cup \mathfrak{S}\right)$ is of type-Bl at the point $x^{\prime} \in X^{\prime}$ if there exists a neighbourhood $U^{\prime}$ of $x^{\prime}$ such that $\varphi^{-1}\left(U^{\prime}\right) \in \Re$. Then there exists a fundamental system of neighbourhoods of $x^{\prime}$ with the same property. The set of points $x^{\prime} \in X^{\prime}$ at which $\varphi$ is of type- $\mathrm{Bl}$ is obviously open. If $\varphi$ is of type-Bl at $x^{\prime} \in X^{\prime}$ and $U^{\prime}$ is an open subset of $X^{\prime}$ containing $x^{\prime}$ then the map $\varphi^{-1}\left(U^{\prime}\right) \rightarrow U^{\prime}$ induced by $\varphi$ is of type-Bl at $x^{\prime}$. If $\varphi$ is of type-Blat any point of $X^{\prime}$ we say that $\varphi$ is of type-Bl. If $U^{\prime}$ is an open subset of $X^{\prime}$ and $\varphi^{-1}\left(U^{\prime}\right) \in \Re$, then the map $\varphi^{-1}\left(U^{\prime}\right) \rightarrow U^{\prime}$ induced by $\varphi$ is of type-Bl. Any harmonic map of type- $\mathrm{Bl}$ is non-constant on any component of $X$. Indeed such a component belongs to $\mathfrak{N}$ by the definition and this contradicts the fact that the constants are harmonic on it.

Lemma 3.2. Let $\varphi: X \rightarrow X^{\prime}$ be a harmonic map, $X^{\prime} \in \mathfrak{S} \cup \mathfrak{P}, f$ be a real function on $X, \mathcal{S}^{\prime}$ be the set of non-negative superharmonic functions $s^{\prime}$ on $X^{\prime}$ such that $s^{\prime} \circ \varphi \geq f$ and $U^{\prime}$ be an open subset of $X^{\prime}$ such that $\varphi^{-1}\left(U^{\prime}\right)$ is either empty or belongs to $\mathfrak{P}$ and $f \geq \bar{H}_{i}^{p^{-1}\left(U^{\prime}\right)}$. If $\mathscr{S}^{\prime}$ is non-empty, then its greatest lower bound is harmonic on $U^{\prime}$.

Let $f^{\prime}$ be the greatest lower bound of $\mathscr{S}^{\prime}, s^{\prime} \in \mathscr{S}^{\prime}$ and $\bar{s}^{\prime}$ be a non-negative superharmonic function on $X^{\prime}$ greater than $s^{\prime}$ on $X^{\prime}-U^{\prime}$. Then $\bar{s}^{\prime} \in \mathscr{S}^{\prime}$. 
Indeed if $\varphi^{-1}\left(U^{\prime}\right)$ is empty this is obvious. Otherwise $\bar{s}^{\prime} \circ \varphi \in \overline{\mathscr{S}}_{f}^{p-1}\left(U^{\prime}\right)$ and $\bar{s}^{\prime} \circ \varphi \geq \bar{H}_{f}^{\rho^{-1}\left(U^{\prime}\right)} \geq \dot{f}$. It follows

$$
f^{\prime} \leq R_{S^{\prime}}^{X^{\prime}-U^{\prime}}, \quad f^{\prime}=\inf _{s^{\prime} \in \mathscr{\varphi}^{\prime}} R_{S^{\prime}}^{X^{\prime}-U^{\prime}} .
$$

Since $\left\{R_{s^{\prime}}^{X^{\prime}-U^{\prime}} \mid s^{\prime} \in \mathscr{S}^{\prime}\right\}$ is a lower directed set of harmonic functions on $U^{\prime}, f^{\prime}$ is harmonic on $U^{\prime}$.

TheOREM 3.10. Let $\varphi: X \rightarrow X^{\prime}$ be a harmonic map, $X^{\prime} \in \mathfrak{P} \cup \mathscr{S}$. The follow. ing assertions are equivalent:

a) $\varphi$ is of type-Bl;

$b)$ the set of points at which $\varphi$ is not of type-Bl is polar;

c) for any open set $U^{\prime} \subset X^{\prime}$ and any locally bounded potential $p^{\prime}$ on $U^{\prime}, p^{\prime} \circ \varphi$ is a potential on $\varphi^{-1}\left(U^{\prime}\right)$;

d) for any locally bounded function $f^{\prime}$ on $X^{\prime}$ and any open subset $U^{\prime}$ of $X^{\prime}$, $U^{\prime} \in \mathfrak{P}$, we have

$$
\bar{h}_{f^{\prime} \circ p^{\prime}}^{p^{-1}\left(U^{\prime}\right), x} \leq \bar{h}_{f^{\prime}}^{\left(y^{\prime}, x^{\prime}\right.} \circ \varphi
$$

e) there exists a real function $f^{\prime}$ on $X^{\prime}$ such that for any compact set $K^{\prime}$ of $X^{\prime}$

$$
0<\inf _{x^{\prime} \in K^{\prime}} f^{\prime}\left(x^{\prime}\right)
$$

and for any open relatively compact subset $U^{\prime}$ of $X^{\prime}\left(U^{\prime} \in \mathfrak{P}\right)$ we hawe $h_{f^{\prime} 0^{\prime}}^{-1}\left(l^{\prime}, x^{x}\right)=0$.

$a \Longrightarrow b$ is trivial.

$b \Rightarrow c$. Let $U^{\prime}$ be an open subset of $X^{\prime}, p^{\prime}$ be a locally bounded potential on $U^{\prime}$ and $u$ be the greatest harmonic minorant of $p^{\prime} \circ \varphi$ on $\varphi^{-1}\left(U^{\prime}\right)$. Let $\mathscr{S}^{\prime}$ be the set of non-negative superharmonic functions $s^{\prime}$ on $U^{\prime}$ such that $s^{\prime} \circ \varphi \geq u$ and $u^{\prime}$ be the greatest lower bound of $\mathscr{S}^{\prime}$. Let $V^{\prime}$ be a relatively compact open subset of $X^{\prime}, \bar{V}^{\prime} \subset U^{\prime}$, such that $\varphi^{-1}\left(V^{\prime}\right) \in \Re$. Since $u \leq p^{\prime} \circ \varphi$ and $p^{\prime}$ is bounded on $V^{\prime}, u$ is bounded on $\varphi^{-1}\left(V^{\prime}\right)$. Hence either $\varphi^{-1}\left(V^{\prime}\right)$ is empty or by Lemma 2.7, $u=H_{u}^{p^{-1}\left(V^{\prime}\right)}$ on $\varphi^{-1}\left(V^{\prime}\right)$. By the preceding lemma we see that $u^{\prime}$ is harmonic on $V^{\prime} . \quad u^{\prime}$ is therefore harmonic on $U^{\prime}$ with the exception of a closed polar set. From $u^{\prime} \leq p^{\prime}$ it follows that $u^{\prime}$ is locally bounded. Hence it may be extended to a harmonic function on $U^{\prime}$. Being dominated by a potential it vanishes. Hence $u$ is equal to zero and $p^{\prime} \circ \varphi$ is a potential on $\varphi^{-1}\left(U^{\prime}\right)$.

$c \Rightarrow d$. It is sufficient to do the proof for $X^{\prime}$ connected. Suppose firstly $f^{\prime}$ 
continuous with compact carrier $L^{\prime}$ and let $U^{\prime}$ be an open subset of $X^{\prime}, U^{\prime} \in \mathfrak{P}$. Let $K^{\prime}$ be a compact subset of $U^{\prime}$ such that $X^{\prime}-K^{\prime} \in \mathfrak{P}$ and let $p^{\prime}$ (resp. $q^{\prime}$ ) be a positive potential on $U^{\prime}$ (resp. $\left.X^{\prime}-K^{\prime}\right)$. Then $p^{\prime} \circ \varphi\left(\right.$ resp. $q^{\prime} \circ \varphi$ ) is a potential on $\varphi^{-1}\left(U^{\prime}\right)$ (resp. $\varphi^{-1}\left(X^{\prime}-K^{\prime}\right)$ ). Let $p$ (resp. $q$ ) be a positive Evans potential on $\varphi^{-1}(U)$ (resp. $\varphi^{-1}\left(X^{\prime}-K^{\prime}\right)$ ) associated with $p^{\prime} \circ \varphi$ (resp. $q^{\prime} \circ \varphi$ ). Obviously $p+q$ (resp. $p$ ) converges to infinite at the Alexandroff point of $X$ through $\varphi^{-1}\left(L^{\prime}-K^{\prime}\right)$ (resp. $\left.\varphi^{-1}\left(K^{\prime}\right)\right)$. Let $\underline{s} \in \mathscr{\mathscr { P }}_{f^{\prime} 0^{\prime}, I^{\prime}}^{-1}, x$. There exists an $\alpha \geq 1$ such that $\alpha p \geq \underline{s}$ on $\varphi^{-1}\left(K^{\prime}\right)$. The function $\alpha p+q-\underline{s}$ is a hyperharmonic function on $\varphi^{-1}\left(U^{\prime}-K^{\prime}\right)$ non-negative outside a compact set of $X$ and its lower limit at any boundary point of $\varphi^{-1}\left(U^{\prime}-K^{\prime}\right)$ is non-negative. Hence $\alpha p+q \geq \underline{s}$ on $\varphi^{-1}\left(U^{\prime}-K^{\prime}\right)$. Since $q$ is arbitrary we have $\alpha p \geq \underline{s}$ on $\bar{\varphi}^{-1}\left(U^{\prime}-K^{\prime}\right)$. This inequality being satisfied also on $\varphi^{-1}\left(K^{\prime}\right)$ we get $\alpha p \geq \underline{s}$ on $\varphi^{-1}\left(U^{\prime}\right)$. Since $p$ is a potential on $\varphi^{-1}\left(U^{\prime}\right)$ it follows $\underline{s} \leq 0$ and $h_{f^{\prime} \circ}^{-1\left(U^{\prime}\right), x} \leq 0$. Similarly we get $\bar{h}_{f^{\prime} \circ \varphi^{-1}\left(U^{\prime}\right), x} \geq 0$. By Theorem 3.7, $f^{\prime} \circ \varphi$ is a Wiener function on $\varphi^{-1}\left(U^{\prime}\right)$ and therefore harmonizable on $\varphi^{-1}\left(U^{\prime}\right)$. By Lemma 2.9 a) we see that $f^{\prime} \circ \varphi$ is harmonizable with respect to $\left(\varphi^{-1}\left(U^{\prime}\right), X\right)$ and we get $h_{f^{\prime} o^{\prime}\left(U^{\prime}\right), x}^{q^{\prime}}=0$.

Let now $f^{\prime}$ be as in the theorem and $s^{\prime} \in \overline{\mathscr{W}}_{f^{\prime}}^{U^{\prime}}, x^{\prime}$. There exists a continuous finite function $f_{0}^{\prime}$ on $X^{\prime}$ with compact carrier such that $f^{\prime}-f_{0}^{\prime} \leq s^{\prime}$ on $U^{\prime}$. Then $\left(f^{\prime}-f_{0}^{\prime}\right) \circ \varphi \leq s^{\prime} \circ \varphi$ on $\varphi^{-1}\left(U^{\prime}\right)$. For any point $y \in \partial \varphi^{-1}\left(U^{\prime}\right)$ we have

$$
\liminf _{x \rightarrow y} s^{\prime} \circ \varphi(x) \geq \liminf _{x^{\prime} \rightarrow p(y)} s^{\prime}\left(x^{\prime}\right) \geq 0
$$

since $\varphi(y) \in \partial U^{\prime}$. Hence $s^{\prime} \circ \varphi \in \overline{\mathscr{W}}_{\left.\left(f^{\prime}-f_{0}^{\prime}\right) 0^{\prime}\right)^{-1},}^{\gamma^{\prime}, x}$

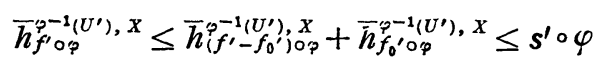

since $\bar{h}_{f_{0}^{\prime} \circ p}^{-1\left(U^{\prime}\right), x}=0$ by the above proof. $s^{\prime}$ being arbitrary we get the required inequality.

$d \Rightarrow \boldsymbol{e}$ is trivial.

$e \Rightarrow a$. Let $U^{\prime}$ be a relatively compact open subset of $X^{\prime}$. Since there exists a positive number $\alpha$ such that $1 \leq \alpha f^{\prime}$ on $U^{\prime}$ we have

$$
\bar{h}_{1}^{p^{-1}\left(U^{\prime}\right), x} \leq \alpha \bar{h}_{f^{\prime \prime} 0^{-1}\left(U^{\prime}\right), x}=0
$$

and $\varphi^{-1}\left(U^{\prime}\right) \in \Re$.

Corollary 3.4. Let $\varphi: X \rightarrow X^{\prime}$ be a harmonic map. If there exists a positive superharmonic function $s^{\prime}$ on $X^{\prime}$ such that $s^{\prime} \circ \varphi$ is a potential then $\varphi$ is of type. $B l$.

The assertion follows from $e \Rightarrow a$. 
Corollary 3.5. Let $\varphi: X^{\prime} X^{\prime}$ be a harmonic map, $X^{\prime}$ connected, and $u^{\prime}$, $v^{\prime}$ be positive harmonic functions on $X^{\prime}$. The following assertions are equivalent:

a) $\varphi$ is of type-Bl and $u^{\prime} \wedge v^{\prime}=0$;

b) $u^{\prime} \circ \varphi \wedge v^{\prime} \circ \varphi=0$.

$a \Longrightarrow b$. Since $u^{\prime} \wedge v^{\prime}=0, \min \left(u^{\prime}, v^{\prime}\right)$ is a potential. Hence

$$
\min \left(u^{\prime} \circ \varphi, v^{\prime} \circ \varphi\right)=\min \left(u^{\prime}, v^{\prime}\right) \circ \varphi
$$

is a potential and we get $u^{\prime} \circ \varphi \wedge v^{\prime} \circ \varphi=0$.

$b \Rightarrow a$. Since $\min \left(u^{\prime}, v^{\prime}\right)$ is a superharmonic function and $\min \left(u^{\prime}, v^{\prime}\right) \circ \varphi$ is a potential, $\varphi$ is of type- $\mathrm{Bl}$ by the preceding corollary. On the other hand $\left(u^{\prime} \wedge v^{\prime}\right) \circ \varphi$ vanishes since it is a minorant of $u^{\prime} \circ \varphi \wedge v^{\prime} \circ \varphi$. Hence $u^{\prime} \wedge v^{\prime}=0$.

Corollary 3.6. Let $\varphi: X \rightarrow X^{\prime}$ be a harmonic map and $F^{\prime}$ be the set of points at which $\varphi$ is not of type-Bl. The intersection of $F^{\prime}$ with any open set is either empty or non-polar.

This follows from $b \Rightarrow a$.

Corollary 3.7. If $\varphi: X \rightarrow X^{\prime}$ is of type-Bl and $X^{\prime}$ connected then any closed set $F^{\prime} \subset X^{\prime}-\varphi(X)$ is polar.

Let $U^{\prime}$ be a domain of $X^{\prime}, U^{\prime} \in \mathfrak{P}$ such that $F^{\prime} \cap U^{\prime}$ is non-polar and $U^{\prime} \cap \varphi(X)$ is non-empty. Then there exists a compact non-polar set $K^{\prime} \subset F^{\prime} \cap U^{\prime}$. The function

$$
s^{\prime}=\left(\hat{R}_{1}^{K^{\prime}}\right)_{U}
$$

is a positive potential. Since $\varphi$ is of type- $\mathrm{Bl}, s^{\prime} \circ \varphi$ is a potential on $\varphi^{-1}\left(U^{\prime}\right)$ by $a \Longrightarrow c$. This is a contradiction since it is harmonic.

Corollary 3.8. Let $\varphi: X \rightarrow X^{\prime}, \varphi^{\prime}: X^{\prime} \rightarrow X^{\prime \prime}$ be harmonic maps and $X, X^{\prime}$, $X^{\prime \prime}$ connected. $\varphi^{\prime \circ} \varphi$ is of type-Bl if and only if $\varphi$ and $\varphi^{\prime}$ are of type-Bl.

Let $U^{\prime \prime}$ be an open subset of $X^{\prime \prime}, U^{\prime \prime} \in \mathfrak{P}$, and $p^{\prime \prime}$ be a positive locally bounded potential on $U^{\prime \prime}$. If $\varphi, \varphi^{\prime}$ are of type-Bl then $p^{\prime \prime} \circ \varphi^{\prime}$ and therefore

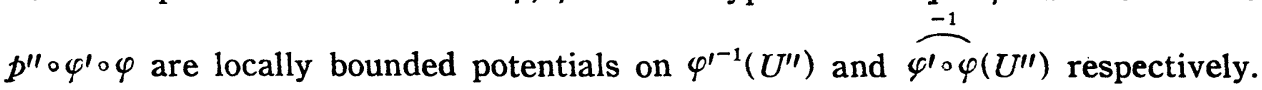
Hence $\varphi^{\prime} \circ \varphi$ is of type-Bl. Conversely if $\varphi^{\prime} \circ \varphi$ is of type- $\mathrm{Bl}$ then $p^{\prime \prime} \circ \varphi^{\prime} \circ \varphi$ is a potential on $\overbrace{\varphi^{\prime} \circ \varphi}^{-1}\left(U^{\prime \prime}\right)$. Let $u^{\prime}$ be the greatest harmonic minorant of $p^{\prime \prime} \circ \varphi$ on $\varphi^{\prime-1}\left(U^{\prime \prime}\right)$. Then $u^{\prime} \circ \varphi$ is a harmonic minorant of $p^{\prime \prime} \circ \varphi^{\prime} \circ \varphi$. It follows $u^{\prime} \circ \varphi=0$, 
$u^{\prime}=0$. Hence $p^{\prime \prime} \circ \varphi^{\prime}$ is a potential and $\varphi, \varphi^{\prime}$ are of type-Bl by Corollary 3.4.

TheOREM 3.11. Let $\varphi: X \rightarrow X^{\prime}$ be a harmonic map of type-Bl, $X \in \mathfrak{P}$, $X^{\prime} \in \mathscr{S}-\mathfrak{P}, X^{\prime}$ connected. If $f^{\prime}$ is a continuous non-negative function on $X^{\prime}$ such that $f^{\prime} \circ \varphi$ is a Wiener function on $X$ then $f^{\prime}$ is harmonic.

Suppose $f^{\prime}$ is non-harmonic and let $u^{\prime}$ be a positive harmonic function on $X^{\prime}$. Let $U_{1}^{\prime}, \ldots, U_{n}^{\prime}$ be a finite covering of $X^{\prime}$ such that $U_{i}^{\prime}$ are open sets with compact boundaries and $U_{i} \in \mathfrak{P}$ for any $i, 1 \leq i \leq m$. There exists for any $i$ a non-negative continuous function $f_{i}^{\prime}$ whose carrier lies in $U_{i}^{\prime}$ such that

$$
u^{\prime}=\sum_{i=1}^{n} f_{i}^{\prime}
$$

Since $X^{\prime} \in \mathfrak{S}-\mathfrak{P}$ we have $\bar{h}_{f_{1}}^{U^{\prime}, X^{\prime}}=0$. Hence by Theorem $3.10(a \Rightarrow d)$

$$
\bar{h}_{f_{i^{\prime} \circ}^{\varphi} \varphi^{-1}\left(l^{\prime} i^{\prime}\right), x} \leq \bar{h}_{f_{i^{\prime}}^{i^{\prime}}, X^{\prime}}^{U^{\prime}} \circ \varphi=0 .
$$

By Theorem $3.8(\mathrm{a} \Rightarrow \mathrm{c}), f_{i}^{\prime} \circ \varphi$ is a Wiener function and therefore harmonizable on $X$. From Corollary 2.2. we deduce that it is a Wiener potential. This is a contradiction since

$$
u^{\prime} \circ \varphi=\sum_{i=1}^{n} f_{i}^{\prime} \circ \varphi
$$

\section{§4. Compactifications}

4.1. We shall suppose from now on $X$ is connected. A compact space $X^{*}$ such that $X$ is a dense subset of $X$, is called a compactification of $X$. Since $X$ is locally compact it is open in $X^{*}$. The closed set $\Delta=X^{*}-X$ is called the ideal boundary of the compactification $X^{*}$.

We shall suppose in this paragraph $X \in \mathfrak{F}$. For any potential $p$ on $X$ we denote by $I_{p}$ the set of points $y \in \Delta$ such that

$$
\liminf _{x \rightarrow y} p(x)=0 \text {. }
$$

We denote further

$$
\Gamma=\bigcap_{p} \Gamma_{p}, \quad \Lambda=\Delta-\Gamma
$$

where $p$ is an arbitrary potential on $X . I$ is compact. If there exists a bounded positive harmonic function on $X$, then $\Gamma_{p}$ is non-empty for any $p$. The family $\left\{\Gamma_{p}\right\}_{p}$ being a filter-basis $\Gamma$ is also non-empty in this case. We call $\Gamma$ the har- 
monic boundary of the compactification $X^{*}$.

Lemma 4.1. If $K$ is a compact set in $\Lambda$, then there exists a continuous potential $p$ on $X$ such that

$$
\lim _{x \rightarrow K} p(x)=\infty
$$

Let $y \in K$. There exists a potential $p_{y}$ on $X$ such that

$$
\liminf _{x \rightarrow y} p_{y}(x)>0 .
$$

Let $G_{y}$ be the set of points $y^{\prime} \in \Delta$ for which

$$
\liminf _{x \rightarrow y^{\prime}} p_{y}(x)>0 .
$$

Since $K$ is compact there exists a finite number of points $y_{1}, \ldots, y_{n}$ on $K$ such that

$$
K \subset \bigcup_{i=1}^{n} G_{y_{i}}
$$

Hence there exists a potential $p^{\prime}$ on $X$ for which

$$
\liminf _{x \rightarrow K} p^{\prime}(x)>0 \text {. }
$$

If $p^{\prime \prime}$ denotes an Evans potential associated with $p^{\prime}$, then

$$
\liminf _{x \rightarrow K} p^{\prime \prime}(x)=\infty \text {. }
$$

There exists a continuous non-negative function $f$ on $X^{*}$, equal to infinite on $K$ not greater than $p^{\prime \prime}$. From

$$
f \leq R_{f}^{X} \leq p^{\prime \prime}
$$

we see that $R_{f}^{X}$ is a continuous potential (see Theorem 3 in [3]) on $X$, converging to infinite at $K$.

$A$ real function $f$ on $X$ is called lower (resp. upper) pseudo-bounded if there exists a potential $p$ such that $f+p$ (resp. $f-p$ ) is lower (resp. upper) bounded, where $\infty-\infty=-\infty+\infty=0$. It is called pseudo-bounded if it is both lower and upper pseudo-bounded. An open non-compact set $U \subset X$ is called M.P.-set if any hyperharmonic function on $U$ is non-negative if its lower limit at the Alexandroff point of $U$ is non-negative. If there exists a superharmonic function on $U$ with positive infimum, $U$ is of type M.P. (by Corollary 1 of [3]). 
Lemma 4.2. Let $U$ be an M.P.-set and $s$ a lower pseudo-bounded hyperharmonic function on $U$. If

$$
\liminf _{x \rightarrow y} s(x) \geq 0
$$

for any point $y$ of the relative boundary of $U$ and for any $y \in \Gamma \cap \bar{U}$, then $s$ is non-negative.

Let $p$ be a potential such that $s_{0}=s+p$ is lower bounded. For any natural number $n$ we denote

$$
K_{n}=\left\{y \in \Delta \cap \bar{U} \mid \liminf _{x \rightarrow y} s_{0}(x) \leq-\frac{1}{n}\right\}
$$

$K_{n}$ is a compact subset of $A$. By the preceding lemma there exists a potential $p_{n}$ on $X$ converging to infinite at $K_{n}$. There exists a sequence $\left\{\varepsilon_{n}\right\}$ of positive numbers such that

$$
p_{0}=\sum_{n=1}^{\infty} \varepsilon_{n} p_{n}
$$

is a potential. For any $y \in \bigcup_{n=1}^{\infty} K_{n}$,

$$
\lim _{x \rightarrow v} p_{0}(x)=\infty
$$

Therefore for any $\varepsilon>0$ the lower limit of $s_{0}+\varepsilon p_{0}$ at the Alexandroff point of $U$ is non-negative. Since $U$ is an M.P.-set, $s_{0}+\varepsilon p_{0}$ is non-negative. $\varepsilon$ being arbitrary, $s_{0}$ is non-negative. We deduce

$$
s \geq h_{s}=h_{s_{0}}+h_{p}=h_{s_{0}} \geq 0 .
$$

Theorem 4.1. (Minimum principle) If $X$ is an M.P.set and $s$ is a lower pseudo-bounded hyperharmonic function on $X$ for which

$$
\liminf _{x \rightarrow \Gamma} s(x) \geq 0 \text {, }
$$

then $s$ is non-negative.

TheоRem 4.2. Let $U$ be an open subset of $X$, whose relative boundary is compact. $\Gamma \cap \bar{U}$ is empty if and only if $U \in \Re$.

If $I \cap \bar{U}$ is empty then $\Delta \cap \bar{U}$ is a compact subset of $A$. There exists therefore a potential $p$ on $X$ converging to $\infty$ at $\Delta \cap \bar{U}$. For any $\varepsilon>0$

$$
\bar{h}_{1}^{r, x} \leq \varepsilon p \text {. }
$$


Hence

$$
\bar{h}_{1}^{U, x}=0 .
$$

Conversely suppose now $\bar{h}_{1}^{U, x}=0$. The function $f$ on $X$ equal to 1 on $U$ and equal to 0 on $X-U$ is then harmonizable with respect to $(X-\partial U, X)$ and $h_{1}^{X-\partial U, X}=0$. Then by Corollary $2.4, f$ is harmonizable on $X$ and $h_{f}^{X}, x=0$. By Lemma 2.2 there exists a potential dominating $f$ outside a compact set. It follows immediately $\Gamma \cap \bar{U} \subset A$.

THEOREM 4.3. Let $X^{*}, X^{* 1}$ be two compactifications of $X$ and $\pi: X^{*} \rightarrow X^{* 1}$ be a continuous map whose restriction on $X$ is the identity. If we denote by $\Gamma^{\prime}$ the harmonic boundary of the compactification $X^{* 1}$ then

$$
\pi(\Gamma)=\Gamma^{\prime}
$$

Let $y \in \Gamma$. For any potential $p$ we have

$$
0 \leq \liminf _{x \rightarrow \pi(y)} p(x) \leq \liminf _{x \rightarrow y} p(x)=0 .
$$

Hence $\pi(\Gamma) \subset \Gamma^{\prime}$. Let now $y^{\prime} \in \Gamma^{\prime}$. For any neighbourhood $U$ of $\pi^{-1}\left(y^{\prime}\right)$ there exists a neighbourhood $U^{\prime}$ of $y^{\prime}$ such that $\pi^{-1}\left(U^{\prime}\right) \subset U$. From here we get

$$
0 \leq \liminf _{x \rightarrow \pi^{-1}\left(y^{\prime}\right)} p(x) \leq \liminf _{x \rightarrow y^{\prime}} p(x)=0 .
$$

Hence $\Gamma_{p} \cap \pi^{-1}\left(y^{\prime}\right)$ is not empty. Since the family $\left\{\Gamma_{p} \cap \pi^{-1}\left(y^{\prime}\right)\right\}_{p}$ is a filter basis $\Gamma \cap \pi^{-1}\left(y^{\prime}\right)$ is not empty and $\Gamma^{\prime} \subset \pi(\Gamma)$.

CoROllaRY 4.1. If for a compatification $X^{*}$ of $X$ the harmonic boundary is not empty then the harmonic boundary of any compactification $X^{*_{1}}$ of $X$ is not empty.

Let $X^{* \prime \prime}$ be the Alexandroff compactification of $X$ and $\pi: X^{*} \rightarrow X^{* \prime \prime}, \pi^{\prime}$ : $X^{* \prime} \rightarrow X^{* \prime \prime}$ be the natural maps. From

$$
\pi^{\prime}\left(\Gamma^{\prime}\right)=\Gamma^{\prime \prime}=\pi(\Gamma)
$$

we see that $\Gamma^{\prime}$ is not empty.

4.2. In order to develop an interesting theory of Dirichlet problem on the ideal boundary it seems necessary to require that $X$ is non-compact, $X \in \mathfrak{P}$, and there exists a superharmonic function on $X$ whose infimum is positive. We shall suppose that $X$ fulfils these conditions. Then $X$ is an M.P.-set. Let 
$f$ be a real function defined on a set which contains $\Delta$. We denote by $\bar{S}_{f}^{x}, x^{*}$ $=\overline{\mathscr{S}}_{f}^{X}=\overline{\mathscr{S}}_{f}\left(\right.$ resp. $\left.: \mathscr{L}_{f}^{X}, X^{*}=\mathscr{L}_{f}^{X}=\mathscr{S}_{f}\right)$ the set of lower bounded hyperharmonic (resp. upper bounded hypoharmonic) functions $s$ on $X$ such that for any $y \in \Delta$

$$
\lim _{x \exists x \rightarrow y} \inf s(x) \geq f(y) \quad \text { (resp. } \lim _{x \exists: x \rightarrow y} \sup _{x} s(x) \leq f(y) \text { ). }
$$

We denote by $\bar{H}_{f}^{X}, x^{*}=\bar{H}_{f}^{X}=\bar{H}_{f}$ (resp. $\underline{H}_{f}^{X}, x^{*}=\underline{H}_{f}^{X}=\underline{H}_{f}$ ) the greatest lower bound of $\overline{\mathscr{S}}_{f}^{X, X^{*}}$ (resp. the least upper bound of $\mathscr{\mathscr { S }}_{f}^{X}, x^{*}$ ). Obviously $\underline{H}_{f} \leq \bar{H}_{f}$ and $\underline{H}_{f}, \bar{H}_{f}$ are either differences of non-negative harmonic functions or identically $\pm \infty$ on $X$.

LEMMA 4.3. Let $A$ be a subset of $X, \chi$ be the characteristic function of $\bar{A} \cap \Delta, f$ be a non-negative pseudo-bounded function on $X$ and $s=\hat{R}_{f}^{A}$. There exists a positive number $\alpha$ such that

$$
h_{s} \leq \alpha \bar{H}_{x} .
$$

There exists a potential $p$ on $X$ and a real number $\alpha^{\prime}$ such that $f \leq p+\alpha^{\prime}$. Let $\alpha>\alpha^{\prime}$ and $\bar{s} \in \overline{\mathscr{S}}_{\alpha \times}$. Then $\bar{s}+p$ dominates $f$ on $A$ outside a compact subset $K$ of $X$. Hence

$$
s \leq \hat{R}_{f}^{K}+\bar{s}+p, \quad h_{s} \leq \bar{s} .
$$

$\bar{s}$ being arbitrary we get

$$
h_{\mathrm{s}} \leq \alpha \bar{H}_{\mathrm{x}} .
$$

If $\underline{H}_{f}, \bar{H}_{f}$ are finite and equal $f$, is called resolutive; their common value is denoted $H_{f}^{X}, x^{*}=H_{f}^{X}=H_{f}$ and is called the solution of Dirichlet problem with $f$ as boundary function.

LEMMA 4.4. Let $f$ be a continuous bounded function on $X^{*} . f$ is resolutive if and only if it is harmonizable on $X$. In this case $h_{f}=H_{f}$.

Obviously $\overline{\mathscr{W}}_{f} \subset \overline{\mathscr{S}}_{f}$. Hence $\bar{H}_{f} \leq \bar{h}_{f}$. Let $s$ be a superharmonic function on $X$ with positive infimum and $\bar{s} \in \overline{\mathscr{S}}_{f}$. Then for any $\varepsilon>0, \bar{s}+\varepsilon s \in \overline{\mathscr{W}}_{f}$ and therefore

$$
\bar{h}_{f} \leq \bar{s}+\varepsilon s .
$$

$\bar{s}$ and $\varepsilon$ being arbitrary we get

$$
\bar{h}_{f} \leq \bar{H}_{f}, \quad \bar{h}_{f}=\bar{H}_{f} .
$$


Similarly it can be proved the relation $\underline{h}_{f}=\underline{H}_{f}$.

A compactification $X^{*}$ of $X$ is called resolutive if any continuous bounded function on $\Delta$ is resolutive.

THEOREM 4.4. The following properties of a compactification $X^{*}$ of $X$ are equivalent:

a) $X^{*}$ is a resolutive compactification;

b) any continuous bounded function on $X^{*}$ is a Wiener function on $X$;

c) for any $x \in X, \omega_{x}^{U *)}$ converges vaguely to a measure on $\Delta$ along the filter (3*.

$a \Longleftrightarrow b$ follows immediately from the preceding lemma.

$b \Longleftrightarrow c$ follows from Theorem 2.8 and Lemma 2.3 .

If $X^{*}$ is a resolutive compactification then for any $x \in X$ the map

$$
f \rightarrow H_{f}(x)
$$

is a positive linear functional on the space of continuous finite functions on $\Delta$. We denote by $\omega_{x}^{x}, x^{*}=\omega_{x}^{X}=\omega_{x}=\omega$ the measure on $\Delta$ such that

$$
\int f d \omega_{x}=H_{f}(x)
$$

for any continuous bounded function $f$ on $\Delta$ and we call it the harmonic measure on $\Delta$ at the point $x$. It follows from Theorem 4.4 and Theorem 2.8 that if $X^{*}$ is a resolutive compactification, $\omega_{x}^{U}$ converges vaguely to $\omega_{x}$ along $\mathcal{B}^{*}$.

$A$ function on $\Delta$ is called $\omega$-integrable if it is $\omega_{x}$-integrable for any $x \in X$. $f$ is $\omega$-integrable if and only if it is $\omega_{x}$-integrable for an $x \in X$ (Axiom 3). If $f$ is $\omega$-integrable, the function $x \rightarrow \int f d \omega_{x}$ is harmonic. $A$ set $A \subset \Delta$ is called $\omega$ measurable if its characteristic function is w-integrable. We denote by $\omega(A)$ the function $x \rightarrow \omega_{x}(A)$ and we call it the harmonic measure of $A$. The set $A$ is called of harmonic measure zero if $\omega(A)$ is identically zero. We say that a property is true almost everywhere on $\Delta$ if it is true at any point of $\Delta$ with the exception of a set of harmonic measure zero.

LEMMA 4.5. Let $X^{*}$ be a resolutive compactification of $X$ and $f$ be a lower

*) For any open set $U \in \Re$ and $x \in U$ we denote by $\omega_{\mathfrak{x}}^{U}$ the harmonic measure on $U$ at the point $x$, i.e. the measure for which $H_{f}^{U}(x)=\int f d \omega_{x}^{U}$ for any continuous finite function $f$ on $\partial U$ with compact carrier. 
bounded lower semicontinuous (resp. upper bounded upper semicontinuous) $\omega$ integrable function on 4 . Then

$$
\int f d \omega=\underline{H}_{f}, \quad\left(\operatorname{resp} . \int f d \omega=\bar{H}_{f}\right) .
$$

Let $f^{\prime}$ be a continuous bounded function on $\Delta, f^{\prime} \leq f$. Then

$$
\begin{gathered}
\underline{H}_{f} \geq H_{f^{\prime}}=\int f^{\prime} d \omega, \\
\underline{H}_{f} \geq \sup _{f^{\prime}} \int f^{\prime} d \omega=\int f d \omega .
\end{gathered}
$$

Let $s \in \mathscr{S}_{f}, f_{0}$ be the function on $\Delta$ equal to

$$
\lim _{x \rightarrow y} \sup s(x)
$$

at any $y \in \Delta$. Then $f_{0}$ is upper semicontinuous, upper bounded and not greater than $f$. There exists a continuous bounded function $f^{\prime}$ on $\Delta$

$$
f_{0} \leq f^{\prime} \leq f
$$

and we have

$$
\begin{aligned}
& s \leq H_{f^{\prime}}=\int f^{\prime} d \omega \leq \int f d \omega, \\
& \underline{H}_{f} \leq \int f d \omega .
\end{aligned}
$$

THEOREM 4.5. Let $X^{*}$ be a resolutive compactification and $f, f^{\prime}$ be $\omega$ integrable functions on 4 . Then

$$
\begin{aligned}
& \int f d \omega \vee \int f^{\prime} d \omega=\int \max \left(f, f^{\prime}\right) d \omega, \\
& \int f d \omega \wedge \int f^{\prime} d \omega=\int \min \left(f, f^{\prime}\right) d \omega .
\end{aligned}
$$

Let $\mathscr{L}_{1}$ (resp. $\mathscr{L}_{2}$ ) be the set of $\omega$-integrable functions $f$ on $\Delta$ for which these relations hold for any continuous bounded (resp. $\omega$-integrable) function $f^{\prime}$ on 4 . Let $f$ be an $\omega$-integrable function and $\left\{f_{n}\right\}$ be a monotone sequence of $\mathscr{L}_{1}$ (resp. $\mathscr{L}_{2}$ ) converging almost everywhere to $f$. From the properties of the integral and of a Riesz space we see that $f$ belongs to $\mathscr{L}_{1}$ (resp. $\mathscr{L}_{2}$ ). Since any continuous bounded function on $\Delta$ belongs to $\mathscr{L}_{1}$ it follows that any $\omega$-integrable function belongs to $\mathscr{L}_{1}$. Hence any continuous bounded function on $\Delta$ belongs to $\mathscr{L}_{2}$ and therefore any $\omega$-integrable function belongs to $\mathscr{L}_{2}$ and 
the proof is complete.

Corollary 4.2. Let $A_{1}, A_{2}$ be w-measurable sets on 4 . Then

$$
\omega\left(A_{1}\right) \vee \omega\left(A_{2}\right)=\omega\left(A_{1} \cup A_{2}\right), \quad \omega\left(A_{1}\right) \wedge \omega\left(A_{2}\right)=\omega\left(A_{1} \cap A_{2}\right) .
$$

CoROllary 4.3. If $U^{*}$ is an open set on a resolutive compactification $X^{*}$, then $\hat{R}_{\omega\left(\Delta \cap U^{*}\right)}^{X-U^{*}}$ is a potential.

Let us denote

$$
f=\omega\left(\Delta \cap U^{*}\right), \quad s=\hat{R}_{f}^{X-U^{*}} .
$$

Since 1 is a Wiener function, $f$ is pseudo-bounded. By Lemma 4.3 and Lemma 4.5 there exists a positive number $\alpha$ such that $h_{s} \leq \alpha \omega\left(\Delta-U^{*}\right)$. Hence

$$
\begin{gathered}
h_{s} \leq \omega\left(\Delta \cap U^{*}\right) \wedge \alpha \omega\left(\Delta-U^{*}\right) \leq \max (\alpha, 1)\left(\omega\left(\Delta \cap U^{*}\right) \wedge \omega\left(\Delta-U^{*}\right)\right) \leq \\
\max (\alpha, 1) \omega\left(\Delta \cap U^{*}-U^{*}\right)=0
\end{gathered}
$$

and $s$ is a potential.

THEOREM 4.6. A compactification $X^{*}$ of $X$ is resolutive if and only if 1 is a Wiener function and one of the following properties are fulfilled:

a) if $u$ is a non-negative pseudo-bounded harnonic function on $X$ and any point of $\Delta$ possesses a neighbourhood $U$ such that $\bar{h}_{u}^{U n X, x}=0$ then $u=0$;

$b$ ) if $f$ is a non-negative pseudo-bounded funstion on $X$ and for any point $y \in \Delta$ there exists a neighbourhood $U$ of $y$ such that $\bar{h}_{f}^{i \cap x, x}=0$ then $\bar{h}_{f}^{x}=0$;

c) if $A, B$ are subsets of $X$ and $\bar{A} \cap \bar{B} \cap \Delta=\phi$ then $\min \left(\hat{R}_{1}{ }^{A}, \hat{R}_{1}^{B}\right)$ is a potential;

d) if $A, B$ are subsets of $\Delta$ and $\bar{A} \cap \bar{B}=\phi$ we have

$$
\bar{H}_{\mathrm{X}_{A} \cup_{B}}=\bar{H}_{\mathrm{X}_{A}}+\bar{H}_{\mathrm{X}_{B}},
$$

where $\%$ denotes the characteristic function of $M$.

We denote by $a^{\prime}$ (resp. $b^{\prime}, c^{\prime}, d^{\prime}$ ) the assertion " $a$ (resp. $b, c, d$ ) and 1 is a Wiener function" and by $e$ the assertion " $X^{*}$ is a resolutive compactification of $X^{\prime \prime}$.

$\boldsymbol{e} \Longrightarrow a^{\prime}$. There exists a finite number of open sets $U_{1}, \ldots, U_{n}$ on $X^{*}$ such that

$$
\Delta \subset \bigcup_{i=1}^{n} U_{i}
$$


and

$$
\bar{h}_{i}^{i n x}=0 .
$$

It follows from Lemma 2.7 and Corollary 1.1

$$
u=\hat{R}_{u}^{X-t^{\prime} i}
$$

and from Lemma 4.3 and Lemma 4.5

$$
\boldsymbol{u} \leq \alpha_{i} \omega\left(\Delta-U_{i}\right)
$$

where $\alpha_{i}$ is a positive number. Hence from Corollary 4.2

$$
u \leq\left(\sup _{i} \alpha_{i}\right) \bigwedge_{i=1}^{n} \omega\left(\Delta-U_{i}\right)=\left(\sup _{i} \alpha_{i}\right) \omega\left(\bigcap_{i=1}^{n}\left(\Delta-U_{i}\right)\right)=0 .
$$

$a^{\prime} \Rightarrow b^{\prime}$. Put $u=\check{h}_{f}$. Since 1 is a Wiener function and $f$ is pseudo-bounded $u$ is finite. By Lemma 2.5 we get

$$
\bar{h}_{u}^{U \cap X}=\bar{h}_{f}^{L \cap X}
$$

for any open set $U$ of $X^{*}$. $u$ is pseudo-bounded and therefore vanishes.

$b^{\prime} \Rightarrow c^{\prime}$. Put $f=\min \left(\hat{R}_{1}^{A}, \hat{R}_{1}^{B}\right)$. For any $y \in \Delta$ there exists a neighbourhood $U$ such that either $U \cap \bar{A}=\phi$ or $U \cap \bar{B}=\phi$. Since $f \leq \hat{R}_{1}^{X-U}$ we get by Corollary 1.1 and Lemma $2.4 \bar{h}_{f}^{\prime \prime} \cap x=0$. Hence $\bar{h}_{f}=0$ and $f$ is a potential.

$c^{\prime} \Longrightarrow d^{\prime}$. Let $U, V$ be open sets of $X^{*}$ such that

$$
\bar{A} \subset U, \quad \bar{B} \subset V, \quad \bar{U} \cap \bar{V}=\phi .
$$

Obviously

$$
\bar{H}_{x_{A}} \leq \hat{R}_{1}^{l \cap n x}, \quad \bar{H}_{\chi_{R}} \leq \hat{R}_{1}^{r \cap x} .
$$

Hence $\min \left(\bar{H}_{\chi_{A}}, \bar{H}_{\chi_{B}}\right)$ is a potential. From

$$
\bar{H}_{\chi_{A}}+\bar{H}_{\chi_{B}}=\min \left(\bar{H}_{x_{A},}, \bar{H}_{\chi_{B}}\right)+\max \left(\bar{H}_{\chi_{A}}, \bar{H}_{\varkappa_{B}}\right),
$$

and Theorem 2.2 we get

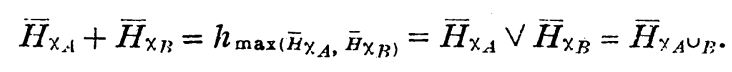

The equality $\bar{H}_{\mathrm{X}_{A} \cup B}=\bar{H}_{\mathrm{X}_{A}} \vee \bar{H}_{\mathrm{X}_{B}}$ can be proved using standard arguments of the Dirichlet problem.

$d^{\prime} \Rightarrow e$. Let $f$ be a continuous bounded function on $X^{*}$ and for any real number $\alpha$, denote

$$
F_{\alpha}=\left\{x \in X^{*} \mid f(x)=\alpha\right\}
$$


By the hypothesis there exists at most a countable set of $\alpha$ such that $\bar{H}_{x_{\alpha}}$ is positive, where $\%_{\alpha}$ denotes the characteristic function of $F_{\alpha} \cap \Delta$. By Lemma 4.3 we see that $\hat{R}_{1}^{r \alpha \cap X}$ is a potential for a dense set of $\alpha$ and by Theorem $2.6 f=$ $f \cdot 1$ is a Wiener function on $X . X^{*}$ is a resolutive compactification by Theorem 4. 4 .

Leмma 4.6. Lef $f$ be a continuous finite function on $X \cap \Gamma$. If $f$ is $a$ Wiener potential on $X$, then $f$ is equal to zero on $\Gamma$. Conversely, if $f$ is pseudobounded and equal to zero on $I$, then $f$ is a Wiener potential on $X$.

The first assertion follows from the fact that $|f|$ is dominated by a potential on $X$ (Lemma 2.2).

Let now $f$ be pseudo-bounded and equal to zero on $I, p_{0}$ be a potential on $X$ such that $|f|-p_{0}$ is upper bounded and $s$ a superharmonic function on $X$ for which

$$
\inf _{x \in X} s(x)=1
$$

For any $\varepsilon>0$ we denote

$$
F_{\varepsilon}=\{y \in X|| f(y) \mid \geq \varepsilon\}, \quad K_{\varepsilon}=\bar{F}_{\varepsilon} \cap \Delta .
$$

Since $K_{\varepsilon}$ is a compact set in $A$ there exists a potential $p$ on $X$ such that

$$
\lim _{x \rightarrow K \varepsilon} p(x)=\infty .
$$

Then $p_{0}+p+\varepsilon s \in \overline{\mathscr{M}}_{|f|}$ and therefore

$$
\bar{h}_{|f|} \leq p_{0}+p+\varepsilon s, \quad \bar{h}_{|f|} \leq \varepsilon s, \quad h_{f}=0 .
$$

THEOREM 4.7. If $X^{*}$ is a resolutive compactification then the carrier of $\omega$ coincides with the harmonic boundary of $X$.

Let $f$ be a continuous bounded function on $\Delta$ equal to zero on $\Gamma$. We extend $f$ to a continuous bounded function on $X^{*}$. From the preceding lemma it follows that $f$ is a Wiener potential on $X$. Hence

$$
\int f d \omega=H_{f}=h_{f}=0
$$

and the carrier of $\omega$ is contained in $\Gamma$.

Let now $U$ be the complement of the carrier of $\omega$ and $f$ be a continuous non-negative bounded function on $\Delta$ whose carrier lies in $U$. We extend $f$ to a continuous bounded function on $X^{*}, f$ is a Wiener function on $X$ and 


$$
h_{f}=H_{f}=\int f d \omega=0
$$

Hence $f$ is la Wiener potential on $X$. From the lemma we deduce that $\Gamma \subset \Delta-U$ and the prdof is completed.

TheOREM 4.8. Let $X^{*}, X^{* \prime}$ be two compactifications of $X$ and $\pi: X^{*} \rightarrow X^{* \prime}$ be a continuious map whose restriction to $X$ is the identical map. If $X^{*}$ is a resolutive compactification then $X^{* \prime}$ is also a resolutive compactification and for any continuous bounded function $f^{\prime}$ on $\Delta^{\prime}=X^{* \prime}-X$ we have

$$
\int f^{\prime} d \omega_{x}^{X, x^{* \prime}}=\int f^{\prime} \circ \pi d \omega_{x}^{X, x^{*}}
$$

We extend $f^{\prime}$ to a continuous bounded function on $X^{* \prime}$. Then $f^{\prime \circ} \pi$ is a continuous bounded function on $X^{*}$. Since $X^{*}$ is a resolutive compactification $f^{\prime}$ is a Wiener function on $X$. Hence $X^{* \prime}$ is a resolutive compactification. The last assertion follows from the obvious relation $\overline{\mathscr{S}}_{f^{\prime}}^{X, X^{* \prime}}=\overline{\mathscr{S}}_{f^{\prime} \circ \pi}^{X, X^{*}}$.

4.3. Let $X^{*}$ be a resolutive compactification. We denote by $H\left(X^{*}\right)$ the set of pseudo-bounded harmonic functions which can be extended continuously on $\Gamma \cup X$.

Lemma 4.7. Let $u \in H\left(X^{*}\right)$ and $u^{*}$ be the limit function of $u$ on $\Gamma$. We have

$$
u=\int u^{*} d \omega
$$

Since $u$ is pseudo-bounded there exists a positive number $\alpha$ and a potential $p$ on $X$ such that $|u| \leq \alpha+p$.

Hence $u^{*}$ is bounded. Let $f$ be a continuous bounded function on $X^{*}$ equal $u^{*}$ on $\Gamma$. Since $u-f$ is pseudo-bounded continuous on $X \cup \Gamma$ and zero on $\Gamma$ it is a Wiener potential on $X$ by Lemma 4.6. Hence

$$
u=h_{f}=H_{f}=\int u^{*} d \omega
$$

Let $X^{*}$ be a resolutive compactification. A puint $y \in \Delta$ is called regular, if for any continuous bounded function $f$ on $\Delta$

$$
\lim _{x \rightarrow ! !} H_{f}(x)=f(y) .
$$


Obviously all points of $A$ are non-regular. If $y$ is regular and $f$ is a bounded function on $\Delta$ continuous at $y$, then

$$
\lim _{x \rightarrow y} \underline{H}_{f}(x)=\lim _{x \rightarrow y} \bar{H}_{f}(x)=f(y) .
$$

THEOREM 4.9. Let $X^{*}$ be a resolutive compactification of $X$. If any point of $\Gamma$ is regular then

$$
\left\{\int f d \omega \mid f \text { is a } \omega \text {-integrable function }\right\}
$$

is the smallest subset of the set of differences of non-negative harmonic functions containing $H\left(X^{*}\right)$ and closed with respect to the monotone limits.

From the above lemma we get

$$
H\left(X^{*}\right)=\left\{\int f d \omega \mid f \text { is a continuous bounded function on } \Delta\right\} .
$$

The theorem follows immediately from this equality.

4.4. Let $X$ be an arbitrary ${ }^{*}$ harmonic space and $X^{*}$ be a compactification of $X$. A subset $A$ of $X$ is called polar if for any domain $U$ of $X, U \in \mathfrak{P}$, there exists a positive superharmonic functions on $U$ such that

$$
\lim _{x \rightarrow A \cap \mathbb{U}} s(x)=\infty \text {. }
$$

For a subset of $X$ this concept coincides with the usual one. If $X \in \mathfrak{P}$ then the compact subsets of $A$ are polar. If $X \in \mathscr{S}-\mathfrak{P}$ and there exists a harmonic function on $X$ with positive infimum then $\Delta$ is a polar set. The subsets of a polar set and the countable union of polar sets are also polar sets. Let $X \in \mathfrak{Y}$, $A \subset \Delta$ and $\psi_{A}$ be the characteristic function of $A ; A$ is polar if and only if $\psi_{A}$ is resolutive and $H_{x_{A}}=0$. Every polar set is therefore of harmonic measure zero, but not any set of harmonic measure zero is polar. This is however true for a set of harmonic measure zero of regular points.

Lemma 4.8. Let $X \in \mathfrak{P} \cup$ S and let $X^{*}$ be a compastification of $X$. A subset $A$ of $X^{*}$ is polar if and only if for any open subset $U$ of $X, U \in \mathfrak{P}$, there exist a compact subset $K$ of $X-U$ and a positive superharmonic function $s$ on $X-K$ such that $K \cap A=\phi$ and

\footnotetext{
*) Not necessarily from $\$$.
} 


$$
\lim _{x \rightarrow A} s(x)=\infty .
$$

The sufficiency and in the case $X \in \mathfrak{P}$ the necessity are trivial. Suppose now $A$ is polar and $X \notin \mathfrak{P}$. Let $U$ be an open subset of $X, U \in \mathfrak{P}$. Then there exists a domain $U^{\prime} \in \mathfrak{P}$ such that $U^{\prime}-U$ is non-polar. There exists by definition a positive superharmonic function $s^{\prime}$ on $U^{\prime}$ infinite on $A \cap U^{\prime}$. If we denote

$$
F_{n}=\left\{x \in U^{\prime}-U \mid s^{\prime}(x) \leq n\right\}
$$

then $F_{n}$ is non-polar for at least an $n$. Let $K$ be a compact non-polar subset of $F_{n}$. Obviously $K \cap A=\phi$. Let $U_{\iota}$ be the components of $X-K$. Since any $U_{\iota} \in \mathfrak{P}$ there exists for any $\mathrm{a}$ positive superharmonic function $s_{\iota}$ on $U_{\iota}$ such that

$$
\lim _{x \rightarrow A \cap \bar{U}_{\ell}} s_{\ell}(x)=\infty
$$

The function $s$ on $X-K$ equal to $s_{\iota}$ on $U_{\iota}$ satisfies the required condition because there are only a finite number of non-relatively compact components of $X-K$.

LеммA 4.9. If $X^{*}$ is a resolutive compactification of $X, U$ an open subset of $X, s$ a positive superharmonic function on $U$ and

$$
A=\left\{y \in \Delta-\overline{X-U} \mid \lim _{x \rightarrow y} s(x)=\infty\right\},
$$

then $A$ is of harmonic measure zero.

Let $\bar{s} \in \overline{\mathscr{S}}_{1}^{X, X^{+}}, \varepsilon>0, s^{\prime} \in \overline{\mathscr{S}}_{\bar{s}}^{U, X}$ and $s_{0}$ be the function on $X$ equal to $\bar{s}$ on $X-U$ and equal to $\min \left(\bar{s}, s^{\prime}+\varepsilon s\right)$ on $U . \quad s_{0}$ is a superharmonic function and belongs to $\overline{\mathscr{S}}_{f_{A}}^{X} X^{*}$, where $f_{A}$ is the characteristic function of $A$ on $\Delta$. We get

$$
\omega(A) \leq s^{\prime}+\varepsilon s
$$

on $U . \quad s^{\prime}$ and $\varepsilon$ being arbitrary it follows $\omega(A) \leq H_{\tilde{s}}^{U}$, and, by Lemma 1.3 and Corollany $1.1, \omega(A)=\hat{R}_{v(A)}^{X-U} \leq \hat{R}_{w(\Delta-\bar{X}-U)}^{X-U}$.

Hence $\omega(A)$ vanishes since $\hat{R}_{\mathrm{w} v(\Delta-\overline{X-U})}^{X-U}$ is a potential by Corollary 4.3.

Let $X^{*}$ be a compactification of $X$ and $\varphi$ be a map of $X$ on a topological space. We denote for any $y \in X^{*}-X$

$$
\varphi^{*}(y)=\bigcap_{U} \overline{\varphi(U \cap X)},
$$

where $U$ runs through the set of neighbourhoods of $y$ in $X^{*}$. 
TheOREM 4.10. Let $\varphi: X \rightarrow X^{\prime}$ be a harmonic non-constant map; $X \in \mathfrak{B}$, $X^{\prime} \in \mathfrak{B} \cup \mathfrak{H}, X^{*}\left(\right.$ resp. $\left.X^{\prime *}\right)$ a compactification of $X$ (resp. $\left.X^{\prime}\right)$. If for a set $A \subset \Delta$ the set

$$
\bigcup_{y \in A} \varphi^{*}(y)
$$

is polar on $X^{\prime *}$ and if $X^{*}$ is a resolutive compactification, then $A$ is of harmonic measure zero.

Denote

$$
A^{\prime}=\bigcup_{y \in A} \varphi^{*}(y)
$$

By Lemma 4.8. there exists a compact subset $K^{\prime}$ of $X^{\prime}$ and a positive superharmonic function $s^{\prime}$ on $X^{\prime}-K^{\prime}$ such that $K^{\prime} \cap A^{\prime}=\phi$ and

$$
\lim _{x^{\prime} \rightarrow A^{\prime}} s^{\prime}\left(x^{\prime}\right)=\infty \text {. }
$$

The function $s^{\prime} \circ \varphi$ is a positive superharmonic function on $\varphi^{-1}\left(X^{\prime}-K^{\prime}\right)$ and

$$
\lim _{x \rightarrow A} s^{\prime} \circ \varphi(x)=\infty .
$$

From the preceding lemma, $A$ is of harmonic measure zero since

$$
A \subset \Delta-\bar{X}^{-1}\left(X^{\prime}-K^{\prime}\right) \text {. }
$$

\section{§. Wiener compactification}

5.1. We shall suppose in this paragraph that $X \in \mathfrak{Y} \cup \mathscr{S}$ and that the function 1 is a Wiener function. There exists a compactification $X^{*}$ such that: a) any Wiener function on $X$ possesses a continuous extension to $X^{*}$; b) the set of these extensions separates $X^{*}$. These two conditions determine uniquely the compactification $X^{*}$ except for a homeomorphism whose restriction to $X$ is the identical map. We shall call this compactification the Wiener compactification of $\mathbf{X}$ and denote it by $X_{\mathscr{W}}^{*} . \quad \Delta_{\mathscr{H}}=X_{\mathscr{H}}^{*}-X$ will be called the Wiener ideal boundary of $X$. Further we denote for $X \in \mathfrak{Y}$ by $\Gamma_{\not / J}$ the harmonic boundary of $X_{\mathscr{V}}^{*}$ and $A_{Z /}=\Delta_{y /}-\Gamma_{\mathscr{W}}$. If misunderstandings will not occur we shall write simply $X^{*}, \Delta, I, \Lambda$ instead of $X_{\mathscr{Z}}^{*}, \Delta_{\mathscr{Z}}, \Gamma_{\mathscr{Z} /}, \Lambda_{\mathscr{Z}}$ respectively.

Lemma 5.1. Let $f$ be a harmonizable function on $X$ and $A$ be the set of points where $f$ is not continuous. $f$ possesses a limit at any point of $X_{j j}^{*}-\bar{A}$.

We may suppose $f$ is non-negative. Let $y \in X^{*}-\bar{A}$ and $f^{\prime}$ be a continuous 
function on $X^{*}, 0 \leq f^{\prime} \leq 1$, equal to 1 on a neighbourhood of $y$ and equal to 0 on $\bar{A}$. Then for any $\alpha>0, \min \left(f, \alpha f^{\prime}\right)$ is a continuous bounded harmonizable function on $X$. Hence it possesses a limit at $y$. Since $\alpha$ is arbitrary $f$ possesses a limit at $y$.

Let $\mathscr{F}$ denote the set of continuous functions $f$ such that there exists a compact set $K_{f}$ for which $f$ is equal either to 0 or to 1 on any component of $X-K_{f}$. We denote by $X_{\mathscr{f}}^{*}$ the compactification for which any function $f \in \mathscr{F}$ can be extended continuously to $X_{\mathscr{F}}^{*}$ and such that these extended functions separate the points of $X_{\mathscr{H}}^{*} . \quad X_{\mathscr{H}}^{*}$ is uniquely determined except for a homeomorphism which induces the identical map on $X$. It can be characterized also by the following property: $X_{\mathscr{F}}^{*}-X$ is totally disconnected and does not disconnect any domain of $X_{\mathscr{F}}^{*}$.

TheOREM 5.1. There exists a canonic continuous map $\pi: X_{\mathscr{W}}^{*} \rightarrow X_{\mathscr{F}}^{*}$ which induces the identical map on $X . \quad\left\{\pi^{-1}(e) \mid e \in X_{\mathscr{F}}^{*}-X\right\}$ is the set of components of $A_{\mathbb{W}}$.

It is sufficient to prove that any function $f \in \mathscr{F}$ is a Wiener function. This follows from Corollary 2.5.

Theorem 5.2. Let $X, X^{\prime}$ be two harmonic spaces, $K$ (resp. $K^{\prime}$ ) be a compact subset of $X$ (resp. $\left.X^{\prime}\right)$ and $\varphi$ be an one to-one harmonic map: $X-K \rightarrow X^{\prime}-K^{\prime}$ such that $\varphi(x)$ converges to $K^{\prime}$ if $x$ converges to $K$. Then $\varphi$ can be extended to a homeomorphism $X_{\mathscr{W}}^{*}-K \rightarrow X_{\mathscr{W}}^{*}-K^{\prime}$.

This is an immediate consequence of the definitions.

Theorem 5.3. Let $X \in \mathfrak{B}$. A compactification $X^{*}$ of $X$ is resolutive if and only if there exists a continuous map $\pi: X_{\mathscr{W}}^{*} \rightarrow X^{*}$ which reduces to the identiy on $X$.

Let $X^{*}$ be a resolutive compactification of $X$. Then for any continuous bounded function $f$ on $X^{*}, f$ is a Wiener function on $X$ (Theorem 4.4). $f$ can be extended therefore continuously on $X_{\| /}^{*}$ and there exists a continuous map $\pi: X_{\mathbb{W}}^{*} \rightarrow X^{*}$ which reduces to the identity on $X$.

Suppose now that there exists such a map $\pi: X_{\mathscr{W}}^{*} \rightarrow X^{*}$ and let $f$ be a continuous function on $X^{*}$. It is sufficient to prove that $f$ is a Wiener function on $X$ (Theorem 4.4). We observe that $f \circ \pi$ is a continuous bounded function on 
$X_{\mathscr{W}}^{*}$. Since $\mathscr{W}$ is a real vector space and a latice with respect to $\max , \min$, there exists a sequence $\left\{f_{n}\right\}$ of continuous bounded functions on $X_{W}^{*}$, such that $f_{n}$ are Wiener functions on $X$, and $\left\{f_{n}\right\}$ converges uniformly to $f \circ \pi$. Since 1 is a Wiener function $\mathscr{W}$ is closed with respect to the uniform convergence. $f$ is therefore a Wiener function on $X$.

Corollary 5.1. The Wiener compactification is resolutive.

Theorem 5.4. All points of $I_{\mathscr{W}}$ are regular.

Let $f$ be a continuous bounded function on $\Delta$. We denote also by $f$ a continuous bounded extension of $f$ on $X^{*}$. Since $f-h_{f}$ is a Wiener potential it can be extended to a continuous bounded function on $X^{*}$ equal to zero on $\Gamma$. $h_{f}$ can be extended therefore continuously on $X^{*}$ equal to $f$ on $I$. The assertion follows now from the equality $h_{f}=H_{f}$ (Lemma 4.4).

5.2. THEOREM 5.5. Let $f$ be a bounded lower semi-continuous function on $\Gamma_{\mathscr{Y}}$ and $\bar{f}$ the function on $\Gamma_{\mathscr{Y}}$

$$
\vec{f}(x)=\lim _{y \rightarrow x} \sup f(y) .
$$

$\vec{f}$ is continuous and differs from $f$ on a polar set.

Denote by $u$ the function

$$
x \rightarrow \int f d \omega_{x}
$$

$u$ may be extended continuously to $\Delta$. If $g$ is a continuous bounded function on $A, g \leq f$ on $\Gamma$, then $H_{g} \leq u$. Hence $u \geq g$ on $I . g$ being arbitrary we have $u \geq f$ on $I$. Since $u=H_{u}, u$ differs from $f$ on a set of harmonic measure zero, which is therefore polar since all points of $I^{\prime}$ are regular. Obviously $\bar{f} \leq u$. Hence $u-\bar{f}$ is a non-negative lower semi-continuous function equal to zero almost everywhere on $\Gamma$. Since the carrier of $\omega$ is equal to $\Gamma, u-\bar{f}$ vanishes identically.

Corollary 5.2. Let $G$ be an open subset of $\Gamma_{\mathscr{W}}$. Then $\bar{G}$ is open on $\Gamma_{\mathscr{H}}$ and $\bar{G}-G$ is polar.

COROLLARY 5.3. $I_{Y /}$ is totally disconnected and either finite or non-metrizable.

THEOREM 5.6. Let F be a closed subset of $X . \quad \hat{R}_{1}^{F}$ is a potential if and only 
if $\bar{F} \cap \Delta_{y} \subset A_{y}$.

If $\bar{F} \cap \Delta \subset \Lambda$ there exists a potential $p$ on $X$ such that

$$
\lim _{x \rightarrow \bar{F} \cap \Delta} p(x)=\infty .
$$

Let $K=\{x \in F \mid p(x) \leq 1\} . \quad K$ is a compact set and therefore $\hat{R}_{1}^{K}$ is a potential. From

$$
\hat{R}_{1}^{F} \leq \hat{R}_{1}^{K}+p
$$

we see that $\hat{R}_{1}^{r}$ is a potential.

Let us suppose now $\hat{R}_{1}^{F}$ is a potential. By Corollary 6 of [3] there exists a continuous potential $p_{0}$ on $X, p_{0} \geq 1$ on $F$. Hence $\bar{F} \cap \Delta \subset A$.

Corollary 5.4. If $\{x \in X \mid\{x\}$ is polar $\}$ is dense outside a compact subset of $X$ then $A_{W}$ is dense in $\Delta_{W}$.

Let $y \in \Gamma$, and $\left\{U_{n}\right\}$ be a pseudo-exhaustion of $X$. Since $X-\bigcup_{n=1}^{\infty} U_{n}$ is a polar set $y \notin \overline{X-\bigcup_{n=1}^{\infty} U_{n}}$. Let $U$ be a neighbourhood of $y$ such that $\bar{U} \cap\left(X-\bigcup_{n=1}^{\infty} U_{n}\right)=\phi$. We may suppose that $U \cup\left(U_{n}-\bar{U}_{n-1}\right) \neq \phi$ for any $n$. Let us take $x_{n} \in U \cap$ $\left(U_{n}-\bar{U}_{n-1}\right)$ such that $\left\{x_{n}\right\}$ is polar. The set $\left\{x_{n} \mid n=1,2, \ldots\right\}$ is polar and has no points of accumulation in $X$. Let $y_{0}$ be a point of accumulation of this sequence. $y_{0} \in \Lambda_{Y} \cap \bar{U}$ and the proof is complete.

TheOREM 5.7. If a point $x \in \Delta_{\mathcal{W}}$ possesses a countable fundamental system of neighbourhoods then $X \in \mathfrak{B}, x \in \Gamma_{\mathscr{W}}$ and there exists a neighbourhood $U$ of $x$ such that any point of $U \cap X$ is not polar.

$X \in \Re$, since otherwise $X_{\mathscr{W}}^{*}$ is Stone-Čech compactification. Let $x \in \Gamma$ and $\left\{U_{n}\right\}$ be a fundamental system of neighbourhoods of $x$. Suppose that there exists for any $n$ a polar point $x_{n} \in U_{n} \cap X$. Then the set $A=\left\{x_{n} \mid n \geq 1\right\}$ is polar and $\bar{A} \cap \Delta=\{x\}$. This contradicts Theorem 5.6.

Let now $x \in A, p$ be a potential on $X$ such that

$$
\lim _{y \rightarrow x} p(y)=\infty,
$$

and $\left\{U_{n}\right\}$ a fundamental system of neighbourhoods of $x, \bar{U}_{n+1} \subset U_{n}$. For any $n$ let $f_{n}$ be a continuous function on $X$ such that $\left|f_{n}\right| \leq p$, sup $f_{n}=\dot{1}$, equal with zero outside $U_{n}-\bar{U}_{n+1}$. The function $f=\sum_{n=1}^{\infty} f_{n}$ is a Wiener potential. This 
is a contradiction since it cannot be extended continuously at $x$.

THEOREм 5.8. If $U$ is an open subset of $X$, then $\bar{U}-\partial \bar{U}$ is an open subset of $X_{\mathscr{W}}^{*}$ and if $X \in \mathfrak{P}, \Gamma_{\mathscr{W}} \cap(\bar{U}-\overline{\partial U})$ is closed $^{* 1}$.

Let $y \in \bar{U}-\overline{\partial U}$ and $f$ be a continuous function on $X^{*}, 0 \leq f \leq 1, f(y)=1$, $f$ equal to 0 on $\partial U$. Then $f$ is a Wiener function on $X$. Let $g$ be a function on $X$ equal to $f$ on $U \cup \partial U$ and equal to 0 on $X-(U \cup \partial U)$. By Lemma 2.11, $g$ is a Wiener function. It can be extended therefore continuously on $X^{*}$, and $g(y)=1$. The set $\left\{x \in X^{*} \mid g(x)>\frac{1}{2}\right\}$ is a neighbourhood of $y$ contained in $\bar{U}-\overline{\partial U}$.

Suppose now $X \in \Re$ and denote $u=\omega(\Gamma \cap(\bar{U}-\overline{\partial U}))$. By Corollary 4.3, $\hat{R}_{u}^{X-U}$ is a potential. By Corollary 6 in [3] $R_{u}^{X-U}$ is dominated by a continuous potential $p$. The function $u-p$ can be extended continuously to $X^{*}$ equal to 1 on $\Gamma \cap(\bar{U}-\overline{\partial U})$ and non-positive on $\Gamma \cap \overline{X-U} \supset \Gamma-(\bar{U}-\overline{\partial U})$. Hence $\Gamma \cap$ $(\bar{U}-\overline{\partial U})$ is closed.

COROLlaRY 5.5. If $U$ is an open subset of $X$ then

$$
\bar{U}-\overline{\partial U}=\bar{U}-\overline{X-} \bar{U} \text {. }
$$

Obviously $\bar{U}-\partial \bar{U} \supset \bar{U}-\bar{X}-U$. Since $\bar{U}-\partial \bar{U}$ is open and $(\bar{U}-\partial U) \cap(X-U)$ $=\phi$ we have $(\bar{U}-\overline{\partial U}) \cap \overline{X-U}=\phi, \bar{U}-\overline{\partial U} \subset \bar{U}-\overline{X-U}$.

CoROLlaRY 5.6. If $U^{*}$ is an open connected set of $X_{\mathscr{W}}^{*}$ then $U^{*} \cap X$ is connected.

Otherwise there would exist two open disjoint non-empty sets $U_{1}, U_{2}, X \in \mathfrak{B}$, $U^{*} \cap X=U_{1} \cup U_{2}$. Since $U^{*}$ is connected $U^{*} \cap \bar{U}_{1} \cap \bar{U}_{2} \neq \phi$. Let $y \in U^{*} \cap \bar{U}_{1} \cap \bar{U}_{2}$. Since $y \notin \partial\left(U^{*} \cap X\right)$ and $\partial U_{i} \subset \partial\left(U^{*} \cap X\right)(i=1,2) y \notin \partial U_{i}$. Hence $y \in\left(\bar{U}_{1}-\partial U_{1}\right)$ $\cap\left(\bar{U}_{2}-\partial U_{2}\right)$. The set $\left(\bar{U}_{1}-\partial \bar{U}_{1}\right) \cap\left(\bar{U}_{2}-\partial \bar{U}_{2}\right)$ being open and non-empty and $X$ being dense in $X^{*}$ we get

$$
U_{1} \cap U_{2}=X \cap\left(\bar{U}_{1}-\bar{\partial} \bar{U}_{1}\right) \cap\left(\bar{U}_{2}-\partial U_{2}\right) \neq \phi,
$$

which is a contradiction.

5. 3. Lemma 5.2. Let $X \in \mathfrak{P}, U$ be an open subset of $X$ and $\left\{U_{c}\right\}$ be the components of $U$. The set

*) $\partial U$ means the boundary of $U$ in $X$ and not in $X^{*}$. 


$$
(\bar{U}-\partial \ddot{U})-\bigcup_{\iota}\left(\bar{U}_{\iota}-\partial U_{\iota}\right)
$$

is a subset of $\Delta_{y /}$ of harmonic measure zero.

Denote by $u$ the harmonic measure of $\Delta \cap(\bar{U}-\partial U)$. By Corollary 4.3. $\hat{R}_{u}^{X-U}$ is a potential and by Corollary 6 in [3] $R_{u}^{X-U}$ is dominated by a continuous potential $p$. The function $s_{\ell}$ equal to $\max (u-p, 0)$ on $U_{l}$ and equal to 0 on $X-U_{c}$ is subharmonic and from its boundary behaviour we see that it minorises $\omega\left(\bar{U}_{\iota}-\partial U_{\iota}\right)$. Hence

$$
\begin{aligned}
& u-p \leq \sum_{\iota} s_{\iota} \leq \sum_{\iota} h_{s_{\iota}} \leq \sum_{\iota} \omega\left(\Delta \cap\left(\bar{U}_{\iota}-\partial U_{\iota}\right)\right)=\omega\left(\Delta \cap\left(\bigcup_{\iota}\left(\bar{U}_{\iota}-\partial U_{\iota}\right)\right)\right), \\
& \omega(\Delta \cap(\bar{U}-\overline{\partial U}))=\omega\left(\Delta \cap\left(\bigcup_{\iota}\left(\bar{U}_{\iota}-\partial \bar{U}_{\iota}\right)\right)\right) .
\end{aligned}
$$

THEOREM 5.9. A point of $\Gamma_{\mathscr{W}}$ is isolated in $\Gamma_{\mathscr{Y}}$ if and only if it has a positive harmonic measure. In this case it possesses a fundamental system of connected neighbourhoods.

If a point of $\Gamma$ is isolated in $\Gamma$ its harmonic measure must be positive since it is open and $\Gamma$ is the carrier of the harmonic measure. Conversely let $y \in I$ be of positive harmonic measure and denote $u=\omega(\{y\})$. $u$ is a positive harmonic function. It can be extended therefore to a continuous function on $\Delta$ equal to 0 on $\Gamma-\{y\}$ and different from 0 at $y$. It follows that $y$ is isolated in $\Gamma$.

Suppose $y \in \Gamma$ has a positive harmonic measure. Let $U^{*}$ be a neighbourhood of $y$ such that $U^{*} \cap \Gamma=\{y\}$. Denote $U=U^{*} \cap X$. Obviously $y \in \bar{U}-\overline{\partial U}$. By the proceding lemma there exists a component $V$ of $U$ such that $y \in \bar{V}-\overline{\partial V}$. By Theorem $5.8, \bar{V}-\partial V$ is a neighbourhood of $y$ and it is obviously connected.

COROLlary 5.7. The dimension of the space of pseudo-bounded harmonic functions on $X$ is equal to $n$ if and only if $\Gamma_{i /}$ consists of exactly $n$ points.

By Lemma 4.7. this space is isomorphic with the space of continuous bounded functions on $\Gamma$.

TheOREM 5.10. A pseudo-bounded harmonic function is minimal if and only if it is proportional to the harmonic measure of a point.

Let $y \in \Gamma$ be of positive harmonic measure and $v$ a harmonic function $0 \leq v \leq \omega(\{y\})$. Since $v$ can be extended continuously to $\Gamma$ we see that $v$ is pro- 
portional to $\omega(\{y\})$.

Let $u$ be a pseudo-bounded minimal function. It can be extended continuously on $\Gamma$ and we have $u=H_{u}^{X}$ (Lemma 4.7). $u$ is equal to zero on $\Gamma$ with the exception of a point $y \in \Gamma$. Indeed on the contrary case we can find two non-negative continuous functions $f_{1}, f_{2}$ on $\Gamma$ not identicaly zero, $f_{1}+f_{2} \leq u$, $\min \left(f_{1}, f_{2}\right)=0$. Since $H_{f_{i}}(i=1,2)$ are positive and proportional to $u$ we get a contradiction since

$$
H_{f_{1}} \wedge H_{f_{2}}=H_{\min \left(f_{1}, f_{2}\right)}=0 .
$$

5.4. Theorem 5.11. Let $X \in \mathfrak{P}$ and $U$ be an open subset of $X$. $U \in \mathfrak{N}$ if and only if $\Gamma_{\mathscr{Y}} \subset X-U$.

Let us denote $\boldsymbol{u}=h_{1}^{x}$. By Lemma 2.5 we have

$$
h_{u}^{U, x}=h_{1}^{U, x} .
$$

Hence $U \in \Re$ if and only if $h_{\mathcal{u}}^{U, x}=0$. By Lemma 2.7 and Corollary 1.1 this is equivalent to the equality

$$
\boldsymbol{u}=\hat{R}_{\mathfrak{u}}^{x-\imath} .
$$

Suppose $I \subset X-U$. Let $s$ be a non-negative hyperharmonic function on $X$, $s \geq u$ on $X-U$. Then by the minimum principle (Theorem 4.1) $s \geq u$ on $X$. Hence

$$
u=\hat{R}_{u}^{X-\iota}
$$

Suppose now

$$
u=\hat{R}_{u}^{x-t}
$$

Then from

$$
u=\omega(\Gamma \cap X-U)+\omega(\Gamma-\overline{X-U})
$$

it follows

$$
\begin{aligned}
& u=\hat{R}_{u}^{X-l^{\prime}}=\hat{R}_{\omega\left(\Gamma \cap \overline{\left.X-U^{\prime}\right)}\right.}^{X-\hat{R}_{w\left(\Gamma-\bar{X}-T^{\prime}\right)}} \leq \omega(\Gamma \cap X-U)+\omega(\Gamma-X-U)=u, \\
& \omega(\Gamma-X-U)=\hat{R}_{\omega(\Gamma-X)}^{X-I T} .
\end{aligned}
$$

Since $\hat{R}_{\omega(\Gamma-\bar{X}-U)}^{X-C^{\circ}}$ is a potential (Corollary 4.3), $\omega(\Gamma-\bar{X}-U)$ vanishes. $\Gamma$ being the carrier of $\omega$ and $\Gamma-X-U$ being open, we have $\Gamma \subset X-U$.

Corollary 5.8. If two open subsets of $X$ coincide outside a compact subset of $X$ then they belong or do not belong simultaneously to $\Re$.

COROLLARY 5.9. If there exist $n$ disjoint open subsets $U_{1}, \ldots, U_{n}$ each 
of which does not belong to $\Re$ and if $\hat{R}_{1}^{X-\bigcup_{i=1}^{n} U_{i}}$ is not a potential, then the dimension of the space of psuedo-bounded harmonic functions on $X$ is at least equal to $n+1$.

The assertion follows from the theorem using Corollary 5.7 and Theorem 5.6.

5.5. Lemma 5.3. Let $F$ be a closed set on $X, f$ be a continuous bounded function on $X$ and $T$ be its carrier on $X$. If the closures of $F$ and $T$ in $X_{W}^{*}$ are disjoint and $f$ is a Wiener function on $X-F$ then $f$ is a Wiener function on $X$. If $X \in \mathfrak{P}$ and $f$ is a Wiener potential on $X-F$ then $f$ is a Wiener potential on $X$.

Suppose firstly $X \in \mathfrak{P}$. There exists a continuous bounded function $f_{0}$ on $X^{*}$ equal to zero on $\bar{F}$ and equal to sup $|f|$ on $T . f_{0}$ is a Wiener function on $X$ and there exists a potential $p$ on $X$ such that $f_{0} \leq h_{f_{0}}+p$. If we denote $s=h_{f_{0}}+p, \hat{R}_{s}^{F}$ is a potential (Lemma 2.6). Since $|f|<s, f$ is a Wiener function (Corollary 2.3). If $f$ is a Wiener potential on $X-F, f$ is a Wiener potential on $X$ by the same corollary.

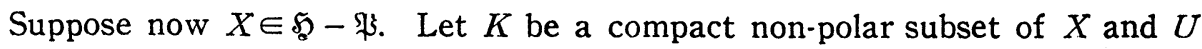
a component of $X-K$. Since $U \in \mathfrak{Y}$ and the closures of $F \cap U$ and $T \cap U$ in $U_{F /}^{*}$ are disjoint, $f$ is a Wiener function on $U$ by the above proof. Hence $f$ is harmonizable on $X-K$ and therefore a Wiener function on $X$.

Theorem 5.12. Let $X^{\prime}$ be a harmonic space and $X$ be a domain of $X^{\prime}$. We denote $F^{\prime}=X^{\prime}-X$ and by $\eta$ the identical map $X \rightarrow X^{\prime}$. $\eta$ possesses a continuous extension to a map $X_{\mathscr{W}}^{*} \rightarrow X_{\mathscr{W}}^{*}$, which we shall denote also by $\eta$. Let us denote further $U^{\prime *}=X_{* \%}^{\prime *}-\bar{F}^{\prime}, U^{*}=\eta^{-1}\left(U^{\prime *}\right)$.

a) The map $U^{*} \rightarrow U^{\prime *}$ defined by $\eta$ is a homeomorphism.

b) If $X^{\prime} \in \mathfrak{P}$ then $\eta\left(\Gamma_{\mathscr{Y}} \cap U^{*}\right)=\Gamma_{\mathscr{y}}^{\prime \prime} \cap U^{\prime *}, \eta\left(A_{\mathscr{Z}} \cap U^{*}\right)=\Lambda_{\mathscr{H}}^{\prime} \cap U^{\prime *}$.

c) If $X^{\prime} \in \mathfrak{P}$ then any $\omega$-measurable set $A \subset \Gamma_{\mathscr{W}} \cap U^{*}$ is of harmonic measure zero (with respect to $X$ ) if and only if $\eta(A)$ is of harmonic measure zero (with respect to $X^{\prime}$ ).

d) $\eta^{-1}\left(\bar{F}^{\prime} \cap \bar{X} \cap \Delta^{\prime}\right)$ is polar.

$\eta$ possesses a continuous extension to a map $X^{*} \rightarrow X^{\prime *}$ since the restriction of any Wiener function on $X^{\prime}$ to $X$ is a Wiener function on $X$.

Let $x, y$ be two different points of $U^{*}$ and $V$ be a neighbourhood of $x$ on $X^{*}$ such that $\overline{\eta(V)} \cap \bar{F}^{\prime}=\phi$. Let $f$ be a continuous bounded function on $X^{*}$ 
whose carrier lies in $V$, equal to 1 at $x$ and equal to 0 at $y$. Its restriction to $X$ is a Wiener function. Let $f^{\prime}$ be the function on $X^{\prime}$ equal to $f$ on $X$ and equal to 0 on $F^{\prime}$. Since $f^{\prime}$ is continuous, $\eta(V)$ contains its carrier and $\overline{\eta(V)} \cap \bar{F}^{\prime}=\phi, f^{\prime}$ is a Wiener function by the preceding lemma. Hence it may be extended continuously on $X^{\prime *}$. We shall denote equally by $f^{\prime}$ this extension. $f^{\prime \circ} \eta$ is equal to $f$ on $X$ and therefore on $X^{*}$.

a) From

$$
f^{\prime}(\eta(x))=f(x)=1, \quad f^{\prime}(\eta(y))=f(y)=0,
$$

it follows $\eta(x) \neq \eta(y)$. $\eta$ is therefore a one-to-one map on $U^{*}$. From

$$
\left\{x^{\prime} \in X^{\prime *} \mid f^{\prime}\left(x^{\prime}\right) \neq 0\right\}=\eta\left(\left\{x \in X^{*} \mid f(x) \neq 0\right\}\right) \subset \eta(V)
$$

we see that $\eta(V)$ is a neighbourhood of $\eta(x)$ and so $\eta$ induces an homeomorphism $U^{*} \rightarrow U^{*}$.

b) Suppose $x \in A \cap U^{*}$. Then we can take $f$ such that it is a Wiener potential on $X$. By the preceding lemma $f^{\prime}$ is also a Wiener potential on $X^{\prime}$. Since

$$
f^{\prime}(\eta(x))=f(x)=1
$$

it follows $\eta(x) \in \Lambda^{\prime} \cap U^{\prime *}, \eta\left(\Lambda \cap U^{*}\right) \subset \Lambda^{\prime} \cap U^{\prime *}$.

Suppose $\eta(x) \in \Lambda^{\prime} \cap U^{\prime *}$. We may take $V$ such that $\eta(V) \cap \Gamma^{\prime}=\phi$. Then $f^{\prime}$ is a Wiener potential on $X^{\prime}$. From Lemma 2.5 we get $h_{f}^{X, X^{\prime}}=0$ and by Lemma 2.7, $h_{f}^{X, X}=0$. Hence $f$ is a Wiener potential on $X$ and $x \in \Lambda$.

c) If $\eta(A)$ is of harmonic measure zero, it is a polar set since all points of $\Gamma^{\prime}$ are regular. By Theorem 4.10, $A$ is of harmonic measure zero.

If $A$ is of harmonic measure zero, there exists a positive superharmonic function $s$ on $X$ converging to infinite at $A . \quad s^{\circ} \eta^{-1}$ converges to infinite at $\eta(A)$ which is therefore of harmonic measure zero by Lemma 4.9.

d) Suppose firstly $X^{\prime} \in \mathfrak{P}$ and denote by $\%$ the characteristic function of $\eta^{-1}\left(\bar{F}^{\prime} \cap \bar{X} \cap \Delta^{\prime}\right)$. We have to show that

$$
\bar{H}_{X}^{X, X^{*}}=0 .
$$

$\bar{H}_{x}^{X, X^{*}}$ is associated with zero at any boundary point of $X$ with respect to $X^{\prime}$. Hence the function $s^{\prime}$ equal to 0 on $F^{\prime}$ and equal to $\bar{H}_{\chi_{*}}^{X, X^{*}}$ on $X$ is the least upper bound of the set of its continuous subharmonic minorants (Theorem 1.2). Let $\underline{s}^{\prime}$ be a continuous subharmonic minorant of $s^{\prime}$. Since $\underline{s}^{\prime}$ is a Wiener func- 
tion it may be extended continuously on $X^{\prime *}$. Since $\bar{H}_{x}^{X, X^{*}}$ converges to zero at $\Gamma \cap U^{*}, \underline{s}^{\prime}$ is equal to zero on

$$
\left(\bar{F}^{\prime} \cap \Delta^{\prime}\right) \cup \eta\left(\Gamma \cap U^{*}\right) \supset \Gamma^{\prime} .
$$

Since $\underline{s}^{\prime}$ is upper pseudo-bounded it is non-positive by the minimum principle (Theorem 4.1). It follows

$$
\bar{H}_{X}^{X}, X^{*}=0 .
$$

Suppose now $X^{\prime}$ is arbitrary. Let $K$ be a compact non-polar subset of $X$, $V_{1}, \ldots, V_{n}$ the non-relatively compact components of $X-K$ and $V_{i}^{\prime}$ the component of $X^{\prime}-K$ which contains $V_{i}$. The assertion follows using the preceding results for the pairs $\left(V_{i}, V_{i}^{\prime}\right),\left(V_{i}, X\right),\left(V_{i}^{\prime}, X^{\prime}\right)$.

\section{$\S 6$. Behaviour of harmonic maps on the ideal boundaries}

6.1. All harmonic spaces considered in this paragraph are connected, belong to $\mathfrak{P} \cup \mathfrak{S}$ and the constants are Wiener functions.

A harmonic map $\varphi: X \rightarrow X^{\prime}$ is called a Fatou map if for any bounded Wiener function $f^{\prime}$ on $X^{\prime}, f^{\prime} \circ \varphi$ is a Wiener function on $X$. A harmonic map $X \rightarrow$ $X^{\prime}$ is a Fatou map if and only if it can be extended continuously into a map $X_{\mathscr{W}}^{*} \rightarrow X_{\mathscr{H}}^{*}$. If $\varphi: X \rightarrow X^{\prime}, \varphi^{\prime}: X^{\prime} \rightarrow X^{\prime \prime}$ are Fatou maps then $\varphi^{\prime} \circ \varphi$ is a Fatou map. The inclusion map is a Fatou map.

THEOREM 6.1. Let $\varphi: X \rightarrow X^{\prime}$ be a harmonic map. If $X, X^{\prime} \in \mathfrak{S}-\mathfrak{P}$ or $X, X^{\prime}$ $\in \mathfrak{P}$, then $\varphi$ is a Fatou map. If $X \in \mathfrak{P}$ and $X^{\prime} \in \mathfrak{S}-\mathfrak{P}$ the following conditions are equivalent:

a) $\varphi$ is a Fatou map;

b) either the constants are non-harmonic on $X^{\prime}$ or there exists a continuous non-constant non-negative function $f^{\prime}$ on $X^{\prime}$ such that $f^{\prime} \circ \varphi$ is a Wiener function on $X$;

c) there exists a closed non-polar set $F^{\prime} \subset X^{\prime}$ such that $\hat{R}_{1}^{p^{-1}\left(F^{\prime}\right)}$ is a potential.

This theorem follows from Theorems 3.7, 3.8, 3.9 remarking that $b$ ) asserts that there exists a continuous non-harmonic non-negative function on $X^{\prime}$ such that composed with $\varphi$ it becomes a Wiener function on $X$.

6.2. Lemma 6.1. Let $U$ be an open set in $\Delta_{y /}$ and $\varphi$ be a continuous map of $X$ in a compact space $Y$. If there exists a polar set $A \subset U$ such that $\varphi$ pos. 
sesses a limit at any point of $U-A$, then $\varphi$ possesses a limit at any point of $U$.

Since any compact space can be imbedded into a cube $[0,1]^{J}$ it is sufficient to prove the lemma for a real function $\varphi, 0 \leq \varphi \leq 1$. Let $y \in A$ and $f_{0}$ be a continuous real function on $X^{*}, 0 \leq f_{0} \leq 1$, equal to 1 on a neighbourhood of $y$ and equal to zero on $\Delta-U$. The function $f=\varphi f_{0}$ is continuous on $X$ and possesses limits at any point of $\Delta-A$. Let $K$ be a compact subset of $X$ such that $X-K \in \mathfrak{F}$ and $s$ be a positive superharmonic function on $X-K$ converging to infinite at any point of $A$. Let $\alpha$ be a positive number and denote

$$
V=\{x \in X-K \mid s(x)>\alpha\} .
$$

There exists a real continuous function $g$ on $X^{*}, 0 \leq g \leq 1$, equal to $f$ on $X-V$. $g$ is harmonizable on $X-K$ and

$$
g-\frac{s}{\alpha} \leq f \leq g+\frac{s}{\alpha}
$$

Hence

$$
\bar{h}_{f}^{X-K, X-K}-\underline{h}_{f}^{X-K, X-K} \leq \frac{2 s}{\alpha} .
$$

$\alpha$ being arbitrary $f$ is harmonizable on $X-K$. It is therefore a Wiener function on $X$ and possesses a limit at $y$. Since $f_{0}$ is equal to 1 on a neighbourhood of $y, \varphi$ possesses a limit at $y$.

THEOREM 6.2. If $X \in \mathfrak{P}$, any continuous map of $X$ in a compact space possesses a limit at any point of $\Lambda_{\mathscr{H}}$.

Any point of $A$ possesses a polar neighbourhood.

Lemma 6.2. Let $U^{*}$ be an open subset of $X_{\mathscr{W}}^{*}$. Any Wiener function on $U^{*} \cap X$ can be extended continuously on $U^{*}$.

It is sufficient to prove the assertion for a Wiener function $f$ on $U^{*} \cap X$, $0 \leq f \leq 1$. Let $y \in U^{*} \cap . \Delta$ and $f_{0}$ be a continuous function on $X^{*}, 0 \leq f_{0} \leq 1$, whose carrier lies in $U^{*}$ and equal to 1 on a neighbourhood of $y$. The function $f^{\prime}$ on $X$ equal to $\min \left(f, f_{0}\right)$ on $U^{*} \cap X$ and equal to 0 on $X-U^{*}$ is a Wiener function by Lemma 5.3. Hence it possesses a limit at $y$. Since $f$ is equal to $f^{\prime}$ on a neighbourhood of $y f$ has a limit at $y$.

Theorem 6.3. Let $\varphi: X \rightarrow X^{\prime}$ be a harmonic map, $X \in \mathfrak{P}$. We define $\varphi^{*}$ 
with respect to $X_{\mathscr{Y}}^{* *},{ }^{* 1}$ and denote

$$
\Delta^{\prime \prime \prime}(\varphi)=\left\{y \in \Delta_{; /} \mid \varphi^{*}(y)=X^{\prime}{ }^{*}\right\} .
$$

Then:

a) for any $y \in \Delta_{\mathscr{W}}-\Delta_{\mathscr{W}}(\varphi), \varphi^{*}(y)$ consists of a point;

b) $\Delta_{\mathscr{W}}(\varphi)$ is an open and closed subset of $I_{\mathscr{W}}$;

c) if $U^{*}$ is an open subset of $X_{\mathscr{H}}^{*}$ and $U^{*} \cap \Delta_{H}(\varphi) \neq \phi$ then there exists a component of $U^{*} \cap X$ on which $\varphi$ is not a Fatou map.

We may suppose $X^{\prime} \in \mathfrak{S}-\mathfrak{P}$ since otherwise $\varphi$ is a Fatou map and therefore may be extended continuously on $X^{*}$.

Let $U^{*}$ be an open subset of $X^{*}$. Suppose that the restriction of $\varphi$ to any component of $U^{*} \cap X$ is a Fatou map. Let $f^{\prime}$ be a continuous bounded function on $X^{\prime *}$. Then $f^{\prime} \circ \varphi$ is a Wiener function on $U^{*} \cap X$ and by the preceding lemma it possesses a continuous extension on $U^{*}$. Hence $\varphi$ possesses a continuous extension on $U^{*}$ and the assertion $\mathrm{c}$ ) is proved.

Let $y \in \Delta-\Lambda_{W}(\varphi)$. Then $y$ possesses a neighbourhood $U^{*}$ such that $X^{\prime}-\varphi\left(U^{*} \cap X\right)$ has interior points. By Theorem 6.1 the restriction of $\varphi$ to any component of $U^{*} \cap X$ is a Fatou map. From the preceding considerations we see that $\varphi$ possesses a continuous extension on $U^{*}$. Hence $\varphi^{*}(y)$ consists of a point and $\Delta_{y y}(\varphi)$ is closed. By Theorem 6.2 it is a subset of $\Gamma$. Let us denote $G=\Gamma-\Delta_{\mathscr{H}}(\varphi)$. By Corollary $5.2 \bar{G}$ is open in $\Gamma$ and $\bar{G}-G$ is polar and from Lemma $6.1, \varphi$ possesses a limit at any point of $\bar{G}$. Hence $\bar{G}=G$ and $\Delta_{7 y}(\varphi)$ is an open subset of $I$.

6.3. Let $\varphi: X \rightarrow X^{\prime}$ be a harmonic map. We shall denote also by $\varphi$ its

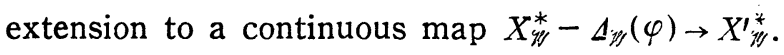

TheOREM 6.4. Let $\varphi: X \rightarrow X^{\prime}$ be a harmonic map, $X \in \mathfrak{B} . \quad X^{\prime} \cap \varphi\left(\Gamma_{Y / J}-\Delta_{i / f}(\varphi)\right)$ is exactly the set of points at which $\varphi$ is not of type-Bl.

Let $x^{\prime} \in X^{\prime} \cap \varphi\left(\Gamma-\Delta_{y}(\varphi)\right)$ and $U^{\prime}$ be a neighbourhood of $x^{\prime}$. There exists a point $y \in \Gamma$ such that $y \notin \overline{X-\varphi^{-1}\left(U^{\prime}\right)}$. From Theorem $5.11, \varphi^{-1}\left(U^{\prime}\right)$ does not belong to $\mathfrak{R} . \varphi$ is therefore not of type- $\mathrm{Bl}$ at $x^{\prime}$.

Let $x^{\prime} \notin X^{\prime} \cap \varphi\left(\Gamma-\Delta_{y}(\varphi)\right)$. Since $X^{\prime} \cap \varphi(\Gamma-\Delta y(\varphi))$ is a closed subset of $X^{\prime}$ there exists a relatively compact neighbourhood $U^{\prime}$ of $x^{\prime}$ such that $\bar{U}^{\prime} \cap \varphi\left(I^{\prime}-\Delta_{Y \prime}(\varphi)\right)=\phi$. If there would exist a point $y \in \Gamma-\bar{X}-\varphi^{-1}\left(U^{\prime}\right)$ then

* see page 43 
$\varphi^{*}(y) \subset \bar{U}^{\prime}$ which is impossible by the preceding theorem. Hence $\Gamma \subset \overline{X-\varphi^{-1}\left(U^{\prime}\right)}$ and $X \cap \varphi^{-1}\left(U^{\prime}\right) \in \Re$ by Theorem 5.11 .

Corollary 6.1. If $X^{\prime} \in \mathscr{S}-\mathfrak{P}, \varphi$ is of type-Bl if and only if $\Gamma_{\mathscr{W}}-\Delta_{y}(\varphi)$ is empty.

If $\Gamma-\Delta_{W}(\varphi)$ is empty, $\varphi$ is type- $B 1$ by the theorem. Suppose now $\varphi$ is of type-Bl. Then $\varphi\left(\Gamma-\Delta_{y j}(\varphi)\right) \subset \Delta^{\prime}$ and the constants are harmonic on $X$ by Theorem 3.11. Then $\Delta^{\prime}$ is polar and $\Gamma-\Delta_{\mathcal{Z}}(\varphi)$ is of harmonic measure zero by Theorem 4.10. Hence $\Gamma-\Delta_{\mathbb{H}}(\varphi)$ is empty since by Theorem 6.3 it is open.

Corollary 6.2. If $X^{\prime} \in \mathfrak{P}, \varphi$ is of type-Bl if and only if

$$
\varphi\left(I_{\mathscr{Y}}\right)=\Gamma_{\mathscr{Y}}^{\prime} .
$$

If $\varphi(\Gamma)=\Gamma^{\prime}$ then $\varphi(\Gamma) \cap X^{\prime}$ is empty and $\varphi$ is of type-Bl. Conversely if $\varphi$ is of type-Bl, then $\varphi(\Gamma) \subset \Delta^{\prime}$. If $\varphi(\Gamma)-\Gamma^{\prime} \neq \phi$ then there would exist an open subset $G$ of $I$ such that $\varphi(G) \subset \Lambda^{\prime}$. This contradicts Theorem 4.10. Hence $\varphi(\Gamma) \subset \Gamma^{\prime}$. Let $f^{\prime}$ be a non-negative bounded continuous function on $X^{\prime *}$ equal to zero on $\varphi(\Gamma)$. There exists a potential $p^{\prime}$ on $X^{\prime}$ such that

$$
h_{f^{\prime}}^{X^{\prime}} \leq f^{\prime}+p^{\prime}
$$

Hence

$$
h_{f}^{X^{\prime}} \circ \varphi \leq f^{\prime} \circ \varphi+p^{\prime} \circ \varphi .
$$

Since $f^{\prime} \circ \varphi$ and $p^{\prime} \circ \varphi$ are Wiener potentials $h_{f^{\prime}}^{x^{\prime}}$ vanishes. It follows $\varphi(\Gamma)=\Gamma^{\prime}$.

Corollary 6.3. If the set of polar points of $\Gamma_{\mathscr{y}}$ is of harmonic measure zero and if any point of $X^{\prime}$ is polar, then $\varphi$ is of type-Bl. If $\varphi$ is also a Fatou map then $X^{\prime} \in \mathfrak{B}$ and the set of poiar points of $\Gamma_{\mathscr{W}}^{\prime}$ is of harmonic measure zero.

Suppose that $\varphi$ is not of type-Bl. Then $X^{\prime} \cap \varphi\left(Y-\Delta_{\mathcal{W}}(\varphi)\right)$ is not empty. $\left(\Gamma-\Delta_{y j}(\varphi)\right) \cap \varphi^{-1}\left(X^{\prime}\right)$ being open and non-empty in $\Gamma$ it contains a non-polar point $x$. This leads to a contradiction since $\varphi(x)$ is polar (Theorem 4.10).

If $\varphi$ is a Fatou map $\Delta_{W}(\varphi)$ is empty. By Corollary $6.1, X^{\prime} \in \mathfrak{P}$. Let $A^{\prime}$ be the set of polar points of $\Gamma^{\prime}$. At any non-polar point of $\Gamma, \omega\left(A^{\prime}\right) \circ \varphi$ converges to zero since the image of such a point does not belong to $A^{\prime}$ by Theorem 4.10. Hence $\omega\left(A^{\prime}\right) \circ \varphi$ and therefore $\omega\left(A^{\prime}\right)$ vanishes.

Lemma 6.3. Let $X, X^{\prime} \in \mathfrak{P}, \varphi: X \rightarrow X^{\prime}$ be an open harmonic map of type$B l$ and $U$ be an open subset of $X$. Then for any $x \in \Gamma_{\mathscr{Z}} \cap(\bar{U}-\bar{X}-\bar{U})$, 
$\varphi(x) \in \Gamma_{\mathscr{y}}^{\prime} \cap\left(\overline{\varphi(U)}-\overline{X^{\prime}-\varphi(U)}\right)$.

We denote

$$
A=\left\{x \in \Gamma \cap(\bar{U}-\overline{X-U}) \mid \varphi(x) \in \overline{X^{\prime}-\varphi(U)}\right\} .
$$

By Corollary 6.2, $\varphi(A) \subset I^{\prime}$ and by Theorem 5.8 and Corollary $5.5, \Gamma^{\prime} \cap X^{\prime}-\varphi(U)$ is an open subset of $\Gamma^{\prime}$. Let us denote

$$
u^{\prime}=\omega\left(\Gamma^{\prime} \cap X^{\prime}-\varphi(U)\right) \text {. }
$$

Then

$$
\boldsymbol{u}^{\prime}=\hat{R}_{\boldsymbol{u}^{\prime}}^{x^{\prime}-\varphi\left(l^{\prime}\right)}, \quad h_{u^{\prime}}^{\varphi\left(l^{\prime}\right), x^{\prime}}=0 .
$$

Hence by Theorem $3.10, a \Rightarrow d$

$$
h_{u^{\prime} \circ g}^{p-1\left(p\left(l^{\prime}\right)\right), x}=0
$$

Since $U \subset \varphi^{-1}(\varphi(U)), h_{u^{\prime} \circ,}^{U, x}$ vanishes. Hence $u^{\prime} \circ \varphi=\hat{R}_{u^{\prime} \circ{ }^{\prime} \gamma}^{X-U}$. From here and from Lemma 4.3 we see that $u^{\prime} \circ \varphi$ converges to zero at any point of $A$. Hence, $A$ is empty, since $\varphi$ is continuous on $A$ and $u^{\prime}$ converges to 1 at $\varphi(A)$.

TheOREm 6.5. Let $X, X^{\prime} \in \mathfrak{B}$ and $\varphi: X \rightarrow X^{\prime}$ be a harmonic map of type$B l$, such that for any $x^{\prime} \in X^{\prime} X \cap \varphi^{-1}\left(x^{\prime}\right)$ contains at most $n$ points $(n<\infty)$. Then for any $y^{\prime} \in \Gamma^{\prime \prime}, \varphi^{-1}\left(y^{\prime}\right) \cap \Gamma_{\mathscr{W}}$ contains at most $n$ points. $y^{\prime}$ has a positive harmonic measure if and only if all points of $\varphi^{-1}\left(y^{\prime}\right) \cap \Gamma_{Y y}$ have a positive harmonic measure.

Being 0-dimensional $\varphi$ is open by Theorem 3.3. Let $y^{\prime} \in I^{\prime}$ and $y_{1}, \ldots, y_{m}$ be different points of $\varphi^{-1}\left(y^{\prime}\right) \cap \Gamma$. There exists a system of pairwise disjoint open sets $U_{1}^{*}, \ldots, U_{m}^{*}$, such that $y_{i} \in U_{i}^{*}(i=1, \ldots, m)$. By the preceding lemma $y^{\prime} \in \overline{\varphi\left(U_{i}^{*} \cap X\right)}-\overline{X^{\prime}-\varphi\left(U_{i}^{*} \cap X\right)}$ for any $i, 1 \leq i \leq m$. Hence $\bigcap_{i=1}^{n} \varphi\left(U_{i}^{*} \cap X\right)$ is not empty. Let $x^{\prime}$ be a point of this set. We can find for any $i, 1 \leq i \leq m$, a point $x_{i} \in U_{i}^{*} \cap X \cap \varphi^{-1}\left(x^{\prime}\right)$. Hence $\varphi^{-1}\left(x^{\prime}\right)$ contains at least $m$ points and we get $m \leq n$. The last assertion follows from Theorem 4.10 and Theorem 5.9.

CoRollary 6.4. Let $\varphi$ be as in the theorem and ' $u^{\prime}$ be a harmonic minimal function on $X^{\prime}$. There exists harmonic minimal functions $u_{1}, \ldots, u_{k}(k \leq n)$ on $X$ such that

$$
u^{\prime} \circ \varphi=\sum_{i=1}^{k} u_{i}
$$


Let us divide the sheaf of harmonic functions on $X$ (resp. $X^{\prime}$ ) by $u^{\prime} \circ \varphi$ (resp. $\left.u^{\prime}\right) . \varphi$ is also type- $\mathrm{Bl}$ with respect to these new harmonic spaces. The function 1 is harmonic and minimal on $X^{\prime}$ and therefore $\Gamma^{\prime}$ (constructed with respect to the new sheaf) consists of a single point (Theorem 5.10). $\Gamma$ consists of $k \leq n$ points $x_{1}, \ldots, x_{k}$ since $\Gamma \subset \varphi^{-1}\left(\Gamma^{\prime}\right)$ (Corollary 6.2 ). Let $\omega_{i}$ denote the harmonic measure of $\left\{x_{i}\right\}$. We have

$$
1=\sum_{i=1}^{k} \omega_{i}
$$

The required equality follows multiplying this equality by $u^{\prime} \circ \varphi$.

CoRollary 6.5. Let $\varphi$ be as in the theorem. If the dimension of the space of pseudo-bounded harmonic functions on $X^{\prime}$ is equal to $d^{\prime}\left(d^{\prime}<\infty\right)$ then the dimension of the space of pseudo-bounded harmonic functions on $X$ is at most equal to $n d^{\prime}$.

If the dimension of this space is finite there exists a basis formed on minimal functions.

\section{BIBLIOGRAPHY}

[1] N. Boboc, C. Constantinescu, A. Cornea, On the Dirichlet problem in the axiomatic theory of harmonic functions, Nagoya Math. J. 23 (1964), 73-96.

[2] M. Brelot, Lectures on potential theory (part. IV), Tata Institute of Fundamental Research, Bombay 1960.

[3] C. Constantinescu, A. Cornea, On the axiomatic of harmonic functions I. Ann. Inst. Fourier 13 (1963), 373-388.

Academia R.P.R. 